Prepared in cooperation with the National Park Service

\title{
Water-Quality and Flow Data, Chulitna River Basin, Southwest Alaska, October 2009-June 2012
}



Open-File Report 2013-1009 
Cover: Chulitna River at U.S. Geological Survey gaging station 15298040. (Photograph taken by Charles Couvillion, U.S. Geological Survey, May 26, 2010.) 


\section{Water-Quality and Flow Data, Chulitna River Basin, Southwest Alaska, October 2009-June 2012}

By Timothy P. Brabets

Prepared in cooperation with the National Park Service

Open File Report 2013-1009 


\section{U.S. Department of the Interior \\ KEN SALAZAR, Secretary \\ U.S. Geological Survey \\ Marcia K. McNutt, Director}

U.S. Geological Survey, Reston, Virginia: 2013

For more information on the USGS — the Federal source for science about the Earth, its natural and living resources, natural hazards, and the environment, visit http://www.usgs.gov or call 1-888-ASK-USGS.

For an overview of USGS information products, including maps, imagery, and publications, visit http://www.usgs.gov/pubprod

To order this and other USGS information products, visit http://store.usgs.gov

Any use of trade, firm, or product names is for descriptive purposes only and does not imply endorsement by the U.S. Government.

Although this information product, for the most part, is in the public domain, it also may contain copyrighted materials as noted in the text. Permission to reproduce copyrighted items must be secured from the copyright owner.

Suggested citation:

Brabets, T.P., 2013, Water-quality and flow data, Chulitna River basin, Southwest Alaska, October 2009-June 2012:

U.S. Geological Survey Open-File Report 2013-1009, 30 p. 


\section{Contents}

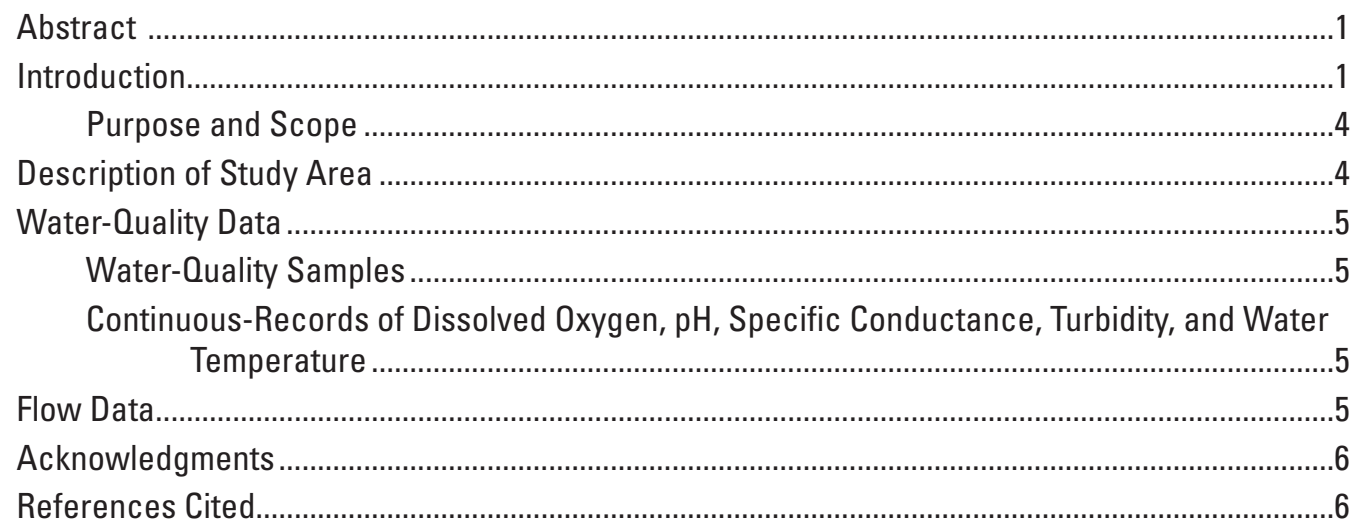

\section{Figures}

1. Map showing location of Lake Clark National Park and Preserve and the Chulitna River basin, southwest Alaska....

2. Digital elevation map showing Lake Clark and Chulitna River basins and major

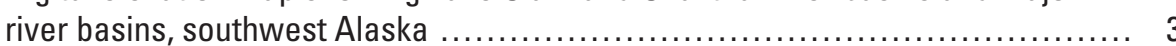

3. Map showing locations of sampling sites, Chulitna River basin, southwest Alaska 


\section{Tables}

1. Description of data-collection sites, Chulitna River basin, southwest Alaska

2. Analyses of water samples collected at USGS streamflow-gaging station 15298040, Chulitna River 5 miles above mouth, near Port Alsworth, Alaska, March 2010-June 2012

3. Summary of standard analytical methods and associated references used in this study

4. Physical field parameters measured at surface-water sites in the Chulitna River basin, Alaska, March 2010-June 2012

5. Concentrations of major ions in water samples collected at USGS streamflow-gaging station 15298040, Chulitna River 5 miles above mouth, near Port Alsworth, Alaska, March 2010-June 2012

6. Dissolved trace-element concentrations in water samples collected at USGS streamflow-gaging station 15298040, Chulitna River 5 miles above mouth, near Port Alsworth, Alaska, March 2010-June 2012

7. Concentrations of nutrients, dissolved organic carbon, and suspended sediment in water samples collected at USGS streamflow-gaging station 15298040, Chulitna River 5 miles above mouth, near Port Alsworth, Alaska, March 2010-June 2012.

8. Results from a blank sample collected at USGS streamflow-gaging station 15298040, Chulitna River 5 miles above mouth, near Port Alsworth, Alaska, August 18, 2010

9. Results from a replicate sample collected at USGS streamflow-gaging station 15298040, Chulitna River 5 miles above mouth, near Port Alsworth, Alaska, March 9, 2011

10. Water temperature data for USGS streamflow-gaging station 15298040 , Chulitna River 5 miles above mouth, near Port Alsworth, Alaska, water years 2010 and 2011.

11. Dissolved oxygen data for USGS streamflow-gaging station 15298040, Chulitna River 5 miles above mouth, near Port Alsworth, Alaska, water years 2010 and 2011.

12. $\mathrm{pH}$ data for USGS streamflow-gaging station 15298040, Chulitna River 5 miles above mouth, near Port Alsworth, Alaska, water years 2010 and 2011

13. Specific conductance data for USGS streamflow-gaging station 15298040 , Chulitna River 5 miles above mouth, near Port Alsworth, Alaska, water years 2010 and 2011

14. Turbidity data for USGS streamflow-gaging station 15298040, Chulitna River 5 miles above mouth, near Port Alsworth, Alaska, water years 2010 and 2011

15. Discharge data for USGS streamflow-gaging station 15298040, Chulitna River 5 miles above mouth, near Point Alsworth, Alaska, water years 2010 and 2011 


\section{Conversion Factors, Datums, and Abbreviations and Acronyms}

Conversion Factors

\begin{tabular}{lcl}
\hline & Multiply & \multicolumn{1}{c}{ By obtain } \\
\hline inch (in.) & Length & \\
inch (in.) & 2.54 & centimeter $(\mathrm{cm})$ \\
foot (ft) & 25.4 & millimeter $(\mathrm{mm})$ \\
mile (mi) & 0.3048 & meter $(\mathrm{m})$ \\
\hline & 1.609 & kilometer $(\mathrm{km})$ \\
\hline ounce (oz) & Weight & \\
pound (lb) & 28.35 & gram $(\mathrm{g})$ \\
\hline & 0.4536 & kilogram $(\mathrm{kg})$ \\
\hline square mile $\left(\mathrm{mi}^{2}\right)$ & Area & \\
square mile $\left(\mathrm{mi}^{2}\right)$ & 259.0 & hectare $($ ha) \\
& 2.590 & square kilometer $\left(\mathrm{km}^{2}\right)$ \\
\hline & Flow rate & \\
\hline cubic foot per second $\left(\mathrm{ft}^{3} / \mathrm{s}\right)$ & 0.02832 & cubic meter per second $\left(\mathrm{m}^{3} / \mathrm{s}\right)$ \\
\hline
\end{tabular}

Temperature in degrees Celsius $\left({ }^{\circ} \mathrm{C}\right)$ may be converted to degrees Fahrenheit $\left({ }^{\circ} \mathrm{F}\right)$ as follows:

$$
{ }^{\circ} \mathrm{F}=\left(1.8 \times{ }^{\circ} \mathrm{C}\right)+32 \text {. }
$$

Specific conductance is given in microsiemens per centimeter at 25 degrees Celsius $(\mu \mathrm{S} / \mathrm{cm}$ at $\left.25^{\circ} \mathrm{C}\right)$.

Concentrations of chemical constituents in water are given either in milligrams per liter (mg/L) or micrograms per liter $(\mu \mathrm{g} / \mathrm{L})$.

Datums

Vertical coordinate information is referenced to the North American Vertical Datum of 1929 (NAVD 29).

Horizontal coordinate information is referenced to the North American Datum of 1927 (NAD 27).

Altitude, as used in this report, refers to distance above the vertical datum.

Abbreviations and Acronyms

FNU formazin Nephelometric units

LACL Lake Clark National Park and Preserve

NFM U.S. Geological Survey, National Field Manual

NWIS U.S. Geological Survey, National Water Information System

USGS U.S. Geological Survey 
This page intentionally left blank. 


\title{
Water-Quality and Flow Data, Chulitna River Basin, Southwest Alaska, October 2009-June 2012
}

\author{
By Timothy P. Brabets
}

\section{Abstract}

The Chulitna River basin in southwest Alaska drains an area of about 1,160 square miles, with the lower 158 square miles of the basin in Lake Clark National Park and Preserve. Water from this basin influences Lake Clark ecosystems that support salmon that, in part, sustain the Bristol Bay fishery. An area of about 391 square miles in the upper part of the Chulitna River basin has been staked for mining development (1,670 claims), and a proposed large scale copper-gold-molybdenum mine (Pebble Mine) lies adjacent to the Chulitna River drainage. The U.S. Geological Survey in cooperation with the National Park Service conducted a water-quality assessment of the Chulitna River from October 2009 to June 2012. Discrete water-quality samples and continuous-records of dissolved oxygen, $\mathrm{pH}$, specific conductance, turbidity, water-stage, and water temperature data were collected from the Chulitna River. In addition, four miscellaneous sites were visited five times during 2010-12 to measure flow and water-quality parameters.

\section{Introduction}

The Chulitna River basin in southwest Alaska is part of the Lake Clark basin in Lake Clark National Park and Preserve (LACL), Alaska (figs. 1-2). The basin supports a variety of aquatic and terrestrial life that depends on high-quality aquatic ecosystems. Fish and wildlife from this area support subsistence harvests by residents of the local communities of Iliamna, Newhalen, Port Alsworth, and Nondalton (Morris, 1986; Fall and others, 1996; Krieg and others, 2005). Fish species present within the basin include northern pike, Arctic grayling and char, burbot, longnose suckers, humpback and round whitefish, least cisco, nine-spine stickleback, slimy sculpin, sockeye salmon, occasional Chinook salmon, and Dolly Varden (Russell, 1980). The Chulitna River provides the only known spawning habitat for humpback whitefish and least cisco in the Lake Clark basin (Woody and Young, 2007). Humpback whitefish are the second most important fish harvested by subsistence users in the region after salmon (Fall and others, 1996; Krieg and others, 2005). Terrestrial wildlife within the drainage include grizzly bear, moose, caribou, and beaver.

The legislative purpose of LACL mandates that the park "....protect the watershed necessary for perpetuation of the red salmon fishery; ... and maintain unimpaired...wild rivers, lakes, waterfalls...in their natural state” (Alaska National Interest Lands Conservation Act, 1980). The number-one natural resource priority for the park is to collect information needed to protect aquatic resources critical for the freshwater part of the salmon life cycle, including baseline information on water-quality and basic habitat characteristics.

Potential mining activity in the region poses a possible threat to the water quality and fisheries habitat in the Chulitna River basin. An area of about $391 \mathrm{mi}^{2}$ in the upper part of Chulitna River basin (fig. 3) has been staked for mining development (1,670 claims), and a proposed large scale copper-gold-molybdenum mine (Pebble Mine) lies adjacent to the Chulitna River drainage (fig. 1). Owners of the Pebble Mine are proceeding with a program of core drilling and environmental sampling, with plans to submit applications for permits by 2013 and to start mining operations several years later. The Pebble Mine deposit is currently estimated at 80.6 billion pounds (lb) of copper, 107.4 million ounces (oz) of gold, and 5.6 billion pounds (lb) of molybdenum (Pebble Partnership, 2012), and has an estimated value ranging from $\$ 300$ to $\$ 500$ billion (American Association for the Advancement of Science, 2011). To date, the Pebble Partnership has spent more than \$100 million to support development of the deposit (Pebble Partnership, 2012). If permitted, the Pebble Mine may include the largest open-pit mine in North America, an underground mine, a 700-ft-high tailings dam, a tailings pond, a mill, a 100-mi transportation corridor with a road and slurry pipeline, a port facility on Cook Inlet, and extensive support facilities (Chambers, 2007; Hauser, 2007; Moran, 2007). 


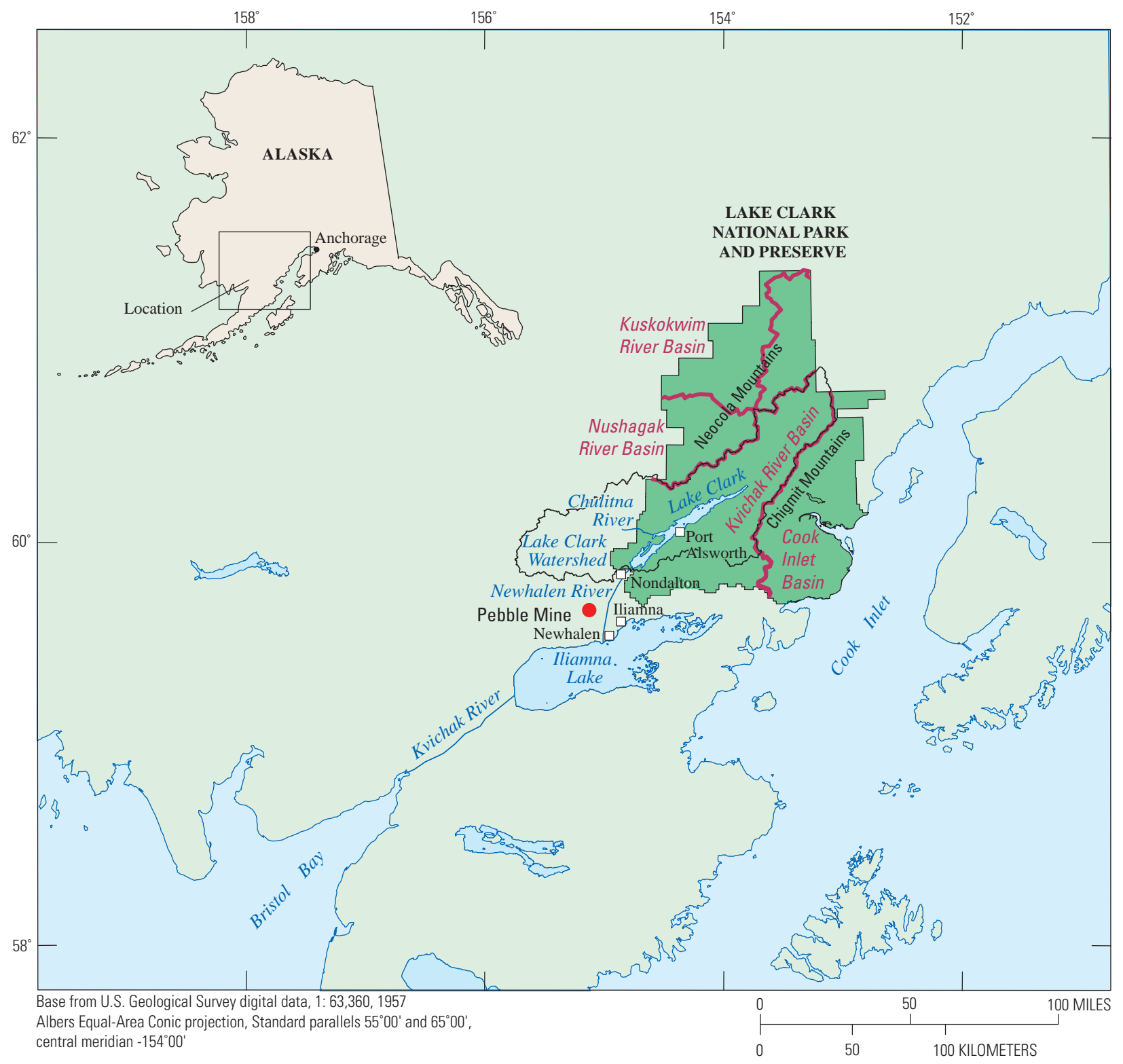

Figure 1. Location of Lake Clark National Park and Preserve and the Chulitna River basin, southwest Alaska. 


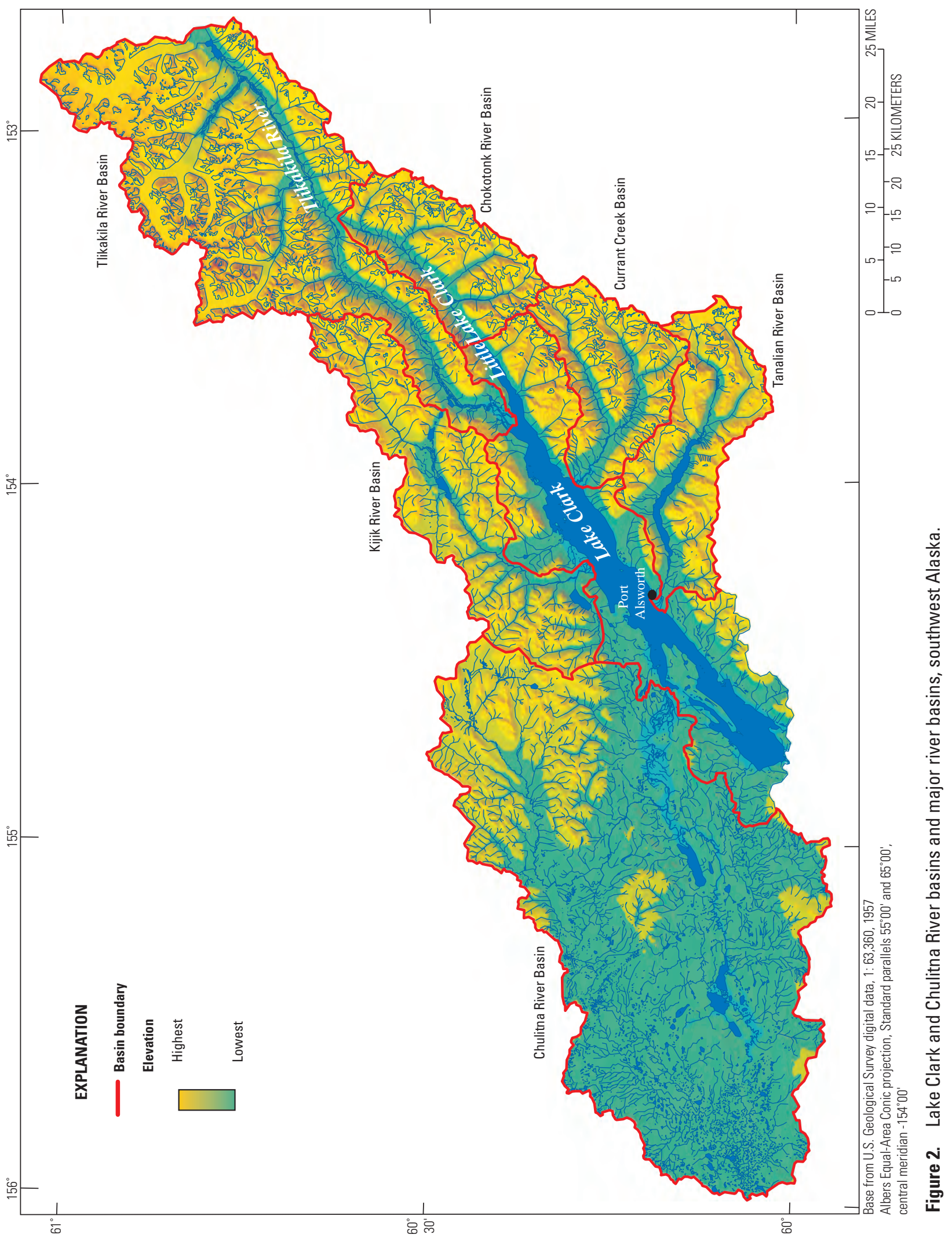




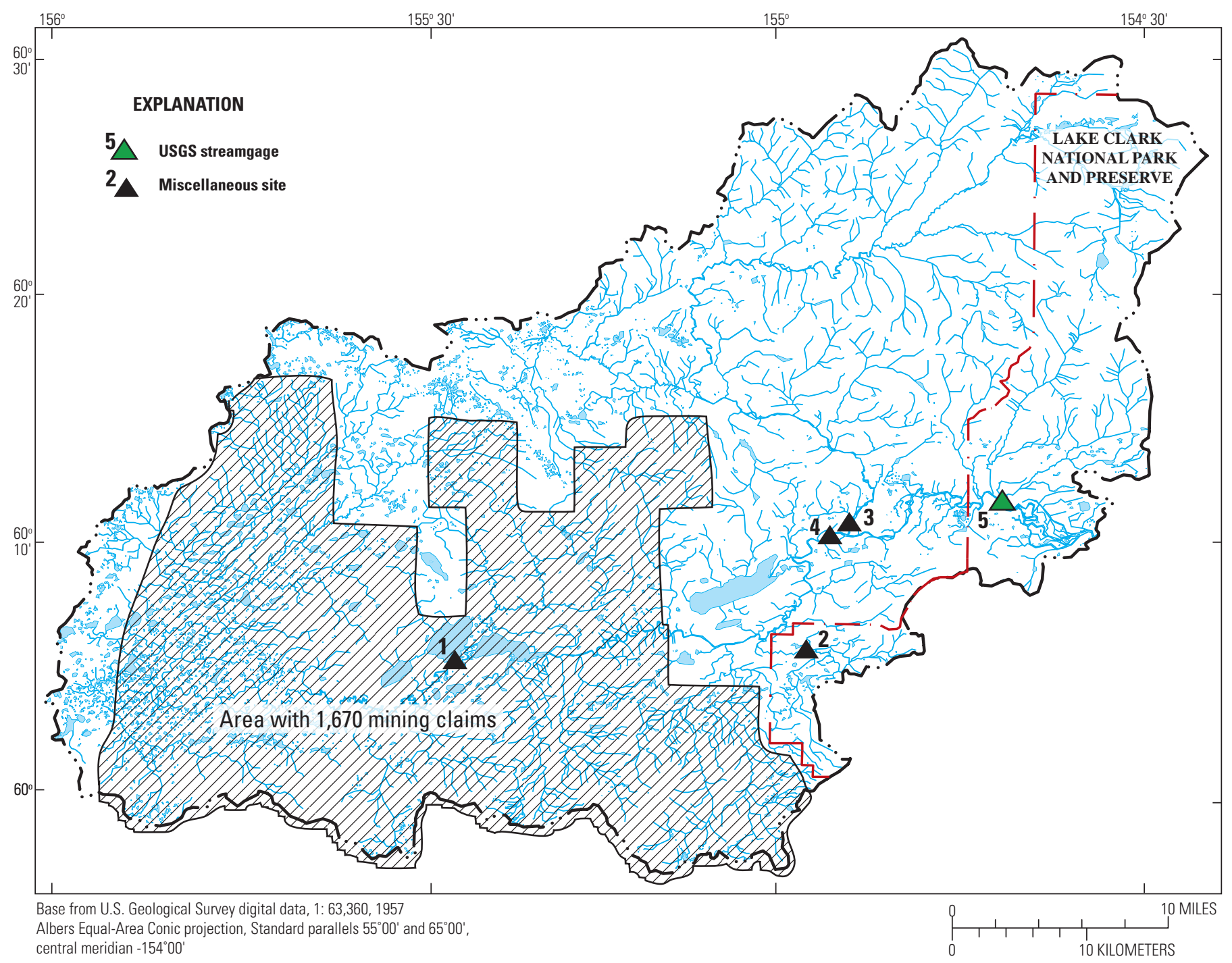

Figure 3. Locations of sampling sites, Chulitna River basin, southwest Alaska.

Although mining currently (2012) is not taking place in the Chulitna River basin, the National Park Service still is responsible for ensuring that waters entering Lake Clark National Park and Preserve remain at a quality level that supports native species. The scale and location of the proposed mining claims could pose a threat to the water quality and fish habitat of the Chulitna River basin and downstream ecosystems because the Chulitna River provides between 20 and 30 percent of the total inflow to Lake Clark (Brabets, 2002).

\section{Purpose and Scope}

This report contains water-quality and flow data collected by the U.S. Geological Survey (USGS) from October 2009 to June 2012 at USGS gaging station 15298040, and at four miscellaneous sites in the Chulitna River basin (fig. 3 and table 1). Water-quality data consist of discrete water samples collected at various times during the hydrologic year at USGS streamgage 15298040, and continuous water-quality monitoring of dissolved oxygen, $\mathrm{pH}$, specific conductance, turbidity, and water temperature at the gaging station. Field parameters were collected at four additional miscellaneous sites. Flow data consist of periodic streamflow measurements and continuous streamflow monitoring. All data are stored in the USGS National Water Information System (NWIS).

\section{Description of Study Area}

Lake Clark National Park and Preserve is in south-central and southwest Alaska (fig. 1). The park is about 6,300 $\mathrm{mi}^{2}$ in area and straddles several river basins. About one-third of the park is in the Cook Inlet basin of south-central Alaska (fig. 1). Streams and rivers in this section of the park flow east into Cook Inlet. The remaining two-thirds of the park 
are in the Kvichak, Kuskokwim, and Nushagak River basins of southwest Alaska. Streams and rivers in the Kvichak and Nushagak River basins flow southwest, eventually entering Bristol Bay. For sockeye salmon (Oncorhynchus nerka), the Kvichak River basin is one of the most productive basins in Alaska. Each year, an estimated 1.5 to 6 million sockeye enter the Lake Clark basin through the Newhalen River to spawn in the many streams in the park.

The Lake Clark basin (fig. 2) drains 2,940 mi . In addition to six major rivers that flow into Lake Clark, there are numerous small glacier-fed streams originating in the Chigmit and Neocola Mountains and clearwater streams originating in the foothills and lowlands that flow into Lake Clark. The Chulitna River basin is $1,160 \mathrm{mi}^{2}$ in area (at the mouth), and is the largest basin in the Lake Clark basin. Mean basin slope is 7 percent, mean annual precipitation is 26 in., average altitude is $1,080 \mathrm{ft}$, no glaciers are present, and lakes occupy an area of $35.5 \mathrm{mi}^{2}$ of the basin (Brabets, 2002). Mesozoic sedimentary rock is the major rock type, and the major soils are spodosols, histosols, and andisols. Low and tall shrubs are the major vegetation. A previous study by Brabets (2002) indicates that the Chulitna River provides 20-30 percent of the inflow to Lake Clark.

\section{Water-Quality Data}

The following sections describe water sample-collection techniques, analytical methods, and flow collection techniques used at the sampling location on the Chulitna River. A summary of site characteristics (latitude, longitude, drainage area, and altitude) is provided in table 1 , and the site locations are shown in figure 3 . All data discussed in this section also are available from the U.S. Geological Survey (2012).

\section{Water-Quality Samples}

Water-quality samples were collected at various times at the USGS streamgage. Samples collected in March 2010, 2011, and 2012 were collected through the river ice. The final sample was collected in June 2012. In total, 12 samples were collected. A broad range of constituents were measured, including field parameters, major ions, dissolved trace elements, nutrients, and suspended sediment (table 2).

Water-quality collection and processing procedures followed USGS National Field Manual (NFM) protocols (U.S. Geological Survey, variously dated). A two-person minimum field team collected samples to reduce the opportunity for contamination of low-concentration analytes, following the protocols of Horowitz and others (1994) and Shelton (1994). Sampling and processing equipment was cleaned prior to each field trip following USGS NFM procedures.
The USGS National Water-Quality Laboratory in Denver, Colorado, analyzed all discrete water samples for dissolved and whole-water constituents using standard USGS methods and quality-assurance practices (Fishman and Friedman, 1989; Patton and Truitt, 1992; Fishman, 1993; Garbarino and others, 2006). A summary of the standard analytical methods used and associated references is provided in table 3 . Analytical results are presented in tables $4-\underline{7}$.

The four miscellaneous sites were visited a total of five times during the data-collection period. Sites were visited in March 2010, 2011, and 2012, a low-flow, ice-covered period, and twice in the summer (July 2010 and August 2011) during average flow or above average flow conditions. At the time of each visit, a discharge measurement was made and water -quality field parameters were measured - dissolved oxygen, $\mathrm{pH}$, specific conductance, turbidity, and water temperature. Data for these sites are presented in table 4.

During the data-collection period, quality-assurance and quality-control protocols were used. A sample containing blank water was collected with the August 18, 2010 sample, and a replicate sample was collected with the March 9, 2011 sample. Results from these 2010 and 2011samples are given in tables 8 and $\underline{9}$, respectively.

\section{Continuous-Records of Dissolved Oxygen, $\mathrm{pH}$, Specific Conductance, Turbidity, and Water Temperature}

At the USGS streamgage 15298040, a Yellow Springs Instrument Model 6920 sonde was installed during the open-water season in water years 2010 and 2011 to collect continuous dissolved oxygen, $\mathrm{pH}$, specific conductance, turbidity, and water temperature data. Data were collected every 15 minutes. This period generally lasted from late May to the end of September. Water temperature also was collected from the stage sensor; therefore, these data are available for the entire water year. Water- quality data were compiled using methods outlined in Wagner and others (2006) and are shown in tables 10-14.

\section{Flow Data}

A sensor was installed in October 2009 at the streamgage to collect continuous stage data. Using methods outlined by Turnispeed and Sauer (2010), a number of discharge measurements were made over a range of flows. A stage-discharge relation was then developed to compute daily discharge at the streamgage. Discharge data for water years 2010 and 2011 are shown in table 15. 


\section{Acknowledgments}

This study was funded by the National Park Service/U.S. Geological Survey Water-Quality Partnership Program. Special thanks go to Dan Young of LACL at Port Alsworth who arranged housing for USGS personnel during sampling trips, boats to access the Chulitna River gaging station, and the use of the park plane to access the miscellaneous sites.

\section{References Cited}

Alaska National Interest Lands Conservation Act, 1980, 16 U.S.C 3101 et seq. (1988), December 2, 1980, Stat. 2371, Pub. L. 96-487.

American Association for the Advancement of Science, 2011, Proposed Pebble Mine has Alaskan community focused on critical science and policy issues: American Association for the Advancement of Science news release, accessed October 22, 2012, at http://www.aaas.org/news/ releases/2011/1018arctic div pebble.shtml.

Brabets, T.P., 2002, Water quality of the Tlikakila River and five major tributaries to Lake Clark, Lake Clark National Park and Preserve, Alaska 1999-2001: U.S. Geological Survey Water-Resources Investigations Report 2002-4127, 29 p. (Also available at http://pubs.er.usgs.gov/publication/ wri024127.)

Brenton, R.W., and Arnett, T.L., 1993, Methods of analysis by the U.S. Geological Survey National Water Quality Laboratory-Determination of dissolved organic carbon by UV-promoted persulfate of oxidation and infrared spectrometry: U.S. Geological Survey Open-File Report 92-480, 12 p. (Also available at http://pubs.er.usgs.gov/ publication/ofr92480.)

Chambers, D.M., 2007, Pebble engineering geologyDiscussion of issues: Center for Science in Public Participation, accessed October 22, 2012, at http:// fish4thefuture.com/pdfs/Pebble $\% 20$ Engineering $\% 20$ Geology\%20-\%20Chambers\%20Sep07.pdf.

Fall, J.A., Chythlook, M.B., Schichnes, J.C., and Morris, J.M., 1996, An overview of the harvest and use of freshwater fish by the communities of the Bristol Bay region, southwest Alaska: Alaska Department of Fish and Game, Division of Subsistence, Technical Paper Series, Technical Paper No. 166, 171 p.

Fishman, M.J., ed., 1993, Methods of analysis by the U.S. Geological Survey National Water-Quality LaboratoryDetermination of inorganic and organic constituents in water and fluvial sediments: U.S. Geological Survey OpenFile Report 93-125, 217 p. (Also available at http://pubs. er.usgs.gov/publication/ofr93125.)
Fishman, M.J., and Friedman, L.C., eds., 1989, Method for determination of inorganic substances in water and fluvial sediments: U.S. Geological Survey Techniques of WaterResources Investigations, book 5, chap. A1, 545 p. (Also available at http://pubs.er.usgs.gov/publication/twri05A1.)

Fishman, M.J., Raese, J.W., Gerlitz, C. N., and Husband, R.A., 1994, U.S. Geological Survey approved inorganic and organic methods for the analysis of water and fluvial sediment, 1954-94: U.S. Geological Survey Open-File Report 94-351, 55 p. (Also available at http://pubs.er.usgs. gov/publication/ofr94351.)

Garbarino, J.R., Kanagy, L.K., and Cree, M.E., 2006, Determination of elements in natural-water, biota, sediment and soil samples using collision-reaction cell inductively coupled plasma-mass spectrometry: U.S. Geological Survey Techniques and Methods, book 5, sec. B, chap. 1, 87 p. (Also available at http://pubs.er.usgs.gov/publication/ tm5B1.)

Guy, H.P., 1969, Laboratory theory and methods for sediment analysis: U.S. Geological Survey Techniques of WaterResources Investigations, book 5, chapter C1, 58 p.

Hauser, W.J., 2007, Potential impacts of the proposed Pebble Mine on fish habitat and fishery resources of Bristol Bay: Fisheries Research and Consulting web site, accessed October 22, 2012, at http://fish4thefuture.com/ pdfs/Pebble $\% 20$ Fish $\% 20$ Habitat $\% 20$ Report $\% 20-\% 20$ Hauser\%20Sep\%2007.pdf.

Horowitz, A.J., Demas, C.R., Fitzgerald, K.K., Miller, T.L., and Rickert, D.A., 1994, U.S. Geological Survey protocol for the collection and processing of surface-water samples for the subsequent determination of inorganic constituents in filtered water: U.S. Geological Survey Open-File report 94-539, 57 p. (Also available at http://pubs.er.usgs.gov/ publication/ofr94539.)

Krieg, T., Chythlook, M.B., Coiley-Kenner, P., Holen, D., Kamletz, K., and Nicholson, H., 2005, Freshwater fish harvest and use in communities of the Kvichak watershed: Juneau, Alaska, Alaska Department of Fish and Game, Division of Subsistence, Technical Paper 297.

Moran, R.E., 2007, Pebble hydrogeology and geochemistry issues: Fisheries Research and Consulting web site accessed October 22, 2012, at http://fish4thefuture.com/pdfs/Moran Hydrogeology_Geochemistry 8 9 07.pdf.

Morris, J.M., 1986, Subsistence production and exchange in the Iliamna Lake region, southwest Alaska, 1982-1983: Juneau, Alaska, Alaska Department of Fish and Game, Division of Subsistence, Technical Paper No. 136, 187 p. 
Patton, C.J., and Truitt, E.P., 1992, Methods of analysis by the U.S. Geological Survey National Water Quality Laboratory-Determination of total phosphorus by a Kjeldahl digestion method and an automated colorimetric finish that includes dialysis: U.S. Geological Survey OpenFile Report 92-146, 39 p. (Also available at http://pubs. er.usgs.gov/publication/ofr92146.)

Patton, C.J., and Truitt, E.P., 2000, Methods of analysis by the U.S. Geological Survey National Water Quality Laboratory-Determination of ammonium plus organic nitrogen by a Kjeldahl digestion method and an automated photometric finish that includes digest cleanup by gas diffusion: U.S. Geological Survey Open-File Report 2000-170, 31 p. (Also available at http://pubs.er.usgs.gov/ publication/ofr00170.)

Pebble Partnership, 2012, Prospecting the future: Pebble Partnership web site accessed October 22, 2012, at http:// www.pebblepartnership.com/project.php.

Russell, R., 1980, A fisheries inventory of waters in the Lake Clark National Monument area: Alaska Department of Fish and Game, 124 p.

Shelton, L.R., 1994, Field guide for collecting and processing stream-water samples for the National Water-Quality Assessment Program: U.S. Geological Survey Open-File Report 94-455, 42 p. (Also available at http://pubs.er.usgs. gov/publication/ofr94455.)
Turnipseed, D.P., and Sauer, V.B., 2010, Discharge measurements at gaging stations-U.S. Geological Survey Techniques and Methods, book 3, chap. A8, 87 p. (Also available at http://pubs.er.usgs.gov/publication/tm3A8.)

U.S. Geological Survey, variously dated, National field manual for the collection of water-quality data: U.S. Geological Survey Techniques of Water-Resources Investigations, book 9, chaps. A1-A9. (Also available at http://pubs.er.usgs. gov/publication/twri09.)

U.S. Geological Survey, 2012, USGS water data for Alaska: U.S. Geological Survey National Water Information System web interface, accessed September 14, 2012, at http:// waterdata.usgs.gov/ak/nwis/.

Wagner, R.J., Boulger, R.W., Oblinger, C.J., and Smith, B.A., 2006, Guidelines and standard procedures for continuous water-quality monitors-Station operation, record computation, and data reporting: U.S. Geological Survey Techniques and Methods, book 1, chap. D3, 51 p. (also available at http://pubs.er.usgs.gov/publication/tm1D3.)

Woody, C.A., and Young, D.Y., 2007, Life history and essential habitats of humpback whitefish in Lake Clark National Park, Kvichak River watershed, Alaska: Anchorage, U.S. Fish and Wildlife Service Office of Subsistence Management, Annual Report for Study FIS 05-403. 
Table 1. Description of data-collection sites, Chulitna River basin, southwest Alaska.

[Site No.: Location of Site No. is shown in figure 3. Abbreviations: USGS, U.S. Geological Survey; $\mathrm{mi}^{2}$, square mile]

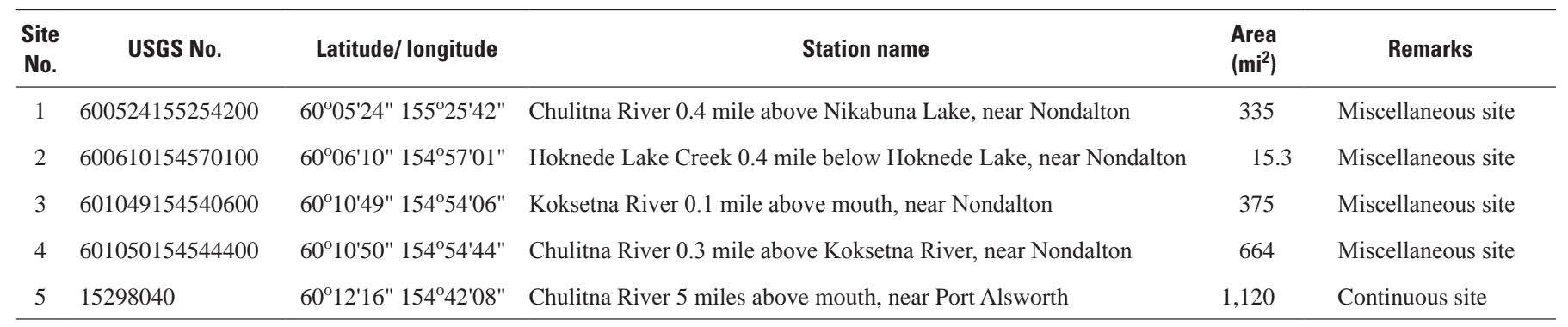

Table 2. Analyses of water samples collected at USGS streamflow-gaging station 15298040, Chulitna River 5 miles above mouth, near Port Alsworth, Alaska, March 2010-June 2012.

[Abbreviations: ${ }^{\circ} \mathrm{C}$, degrees Celsius; $\mu \mathrm{S} / \mathrm{cm}$, microsiemens per centimeter at 25 degrees Celsius; $\mathrm{mg} / \mathrm{L}$, milligram per liter; FNU, Formazin nephelometric unit; $\mathrm{ft}^{3} / \mathrm{s}$, cubic foot per second; $\mu \mathrm{g} / \mathrm{L}$, microgram per liter; mm, millimeter]

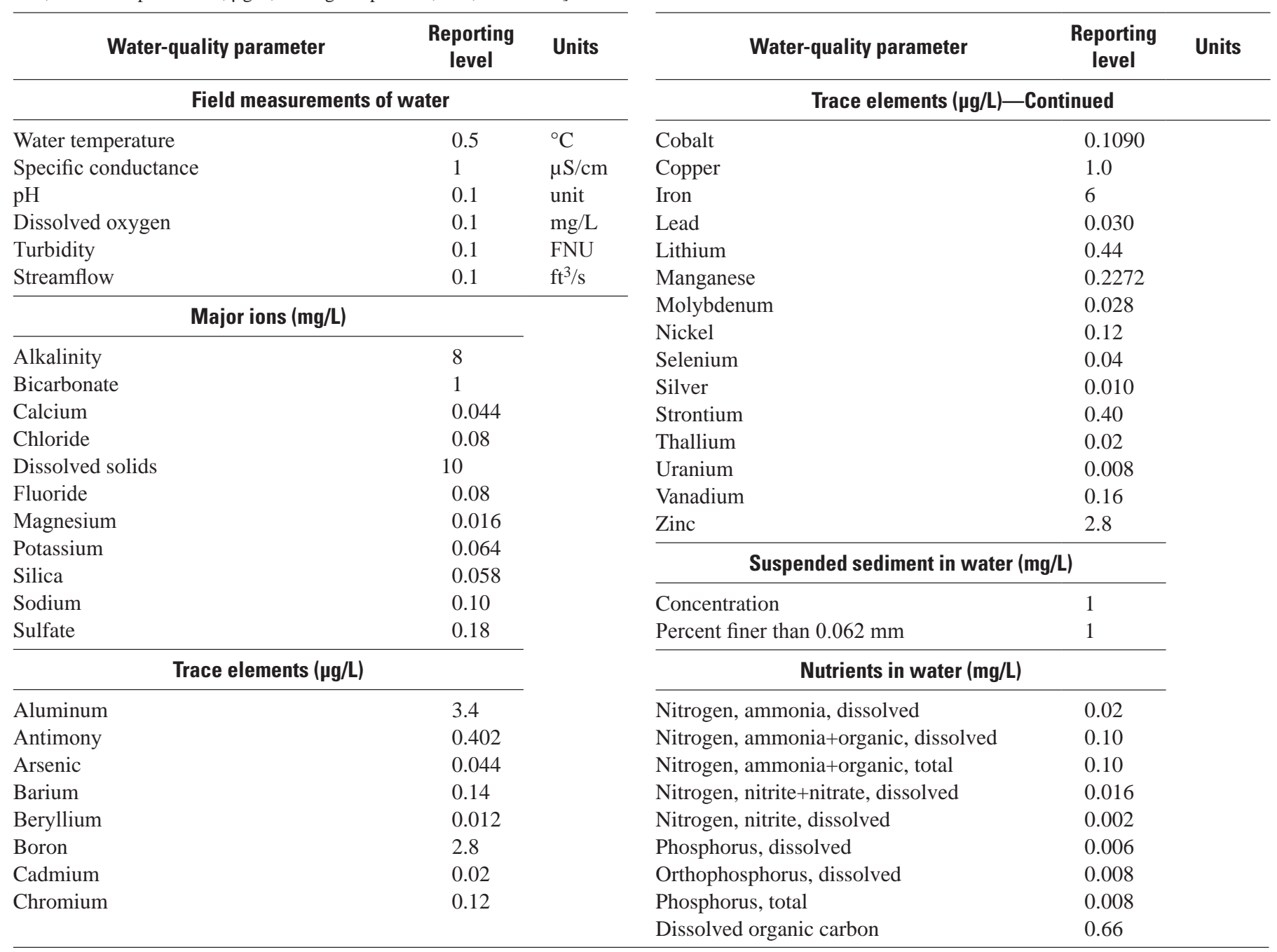


Table 3. Summary of standard analytical methods and associated references used in this study.

[Abbreviations: Dis, dissolved; fet, fixed end-point; tot, total; IT, incremental titration; mm, millimeter]

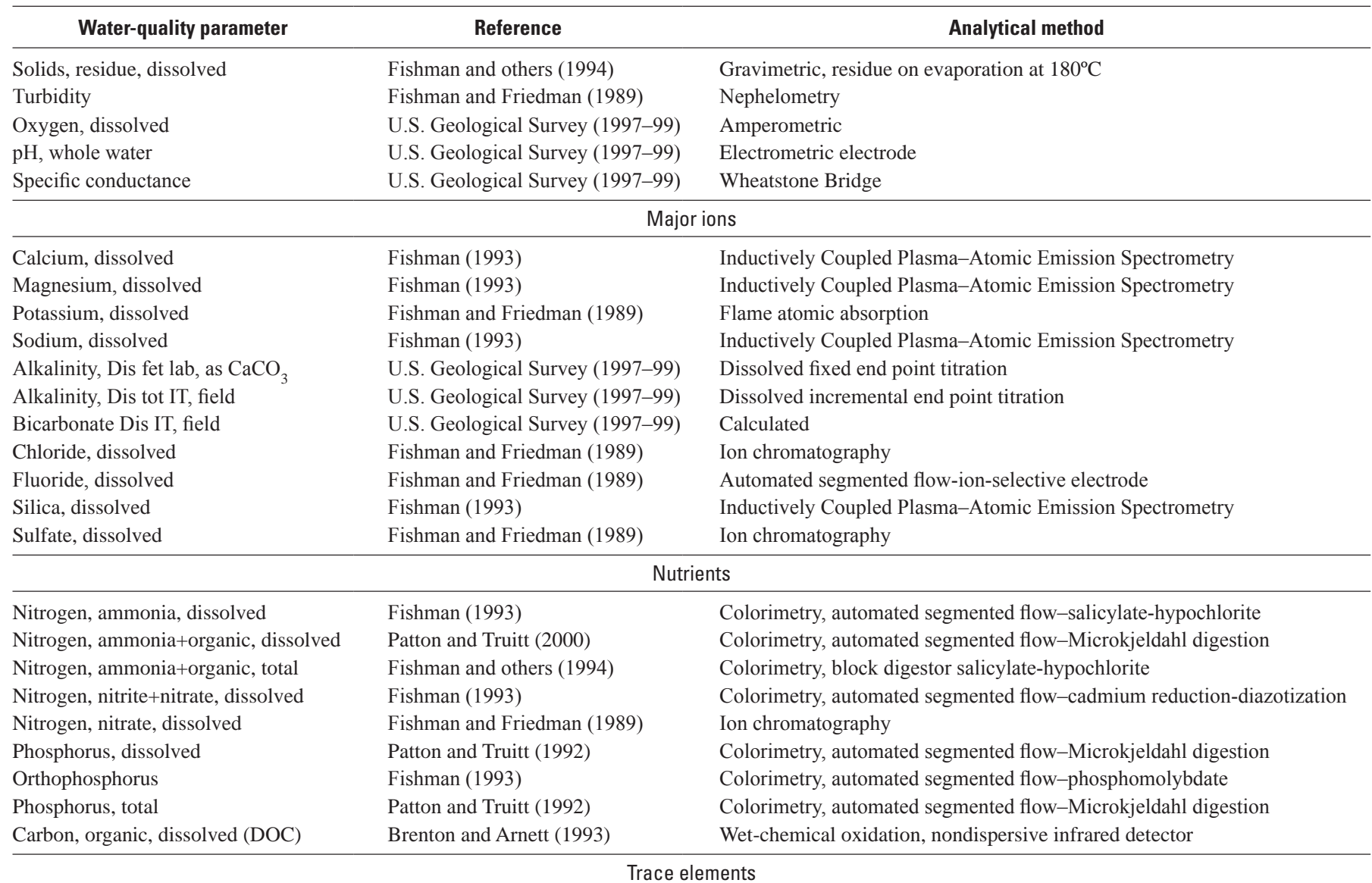

Aluminum, dissolved

Antimony, dissolved

Arsenic, dissolved

Barium, dissolved

Beryllium, dissolved

Boron, dissolved

Cadmium, dissolved

Chromium, dissolved

Cobalt, dissolved

Copper, dissolved

Iron, dissolved

Lead, dissolved

Lithium, dissolved

Manganese, dissolved

Molybdenum, dissolved

Nickel, dissolved

Selenium, dissolved

Silver, dissolved

Strontium, dissolved

Thallium, dissolved

Uranium, dissolved

Vanadium, dissolved

Zinc, dissolved
Garbarino and others (2006)

Garbarino and others (2006)

Garbarino and others (2006)

Garbarino and others (2006)

Garbarino and others (2006)

Garbarino and others (2006)

Garbarino and others (2006)

Garbarino and others (2006)

Garbarino and others (2006)

Garbarino and others (2006)

Fishman (1993)

Garbarino and others (2006)

Garbarino and others (2006)

Garbarino and others (2006)

Garbarino and others (2006)

Garbarino and others (2006)

Garbarino and others (2006)

Garbarino and others (2006)

Garbarino and others (2006)

Garbarino and others (2006)

Garbarino and others (2006)

Garbarino and others (2006)

Garbarino and others (2006)
Inductively Coupled Plasma-Mass Spectrometry Inductively Coupled Plasma-Mass Spectrometry Inductively Coupled Plasma-Mass Spectrometry Inductively Coupled Plasma-Mass Spectrometry Inductively Coupled Plasma-Mass Spectrometry Inductively Coupled Plasma-Mass Spectrometry Inductively Coupled Plasma-Mass Spectrometry Inductively Coupled Plasma-Mass Spectrometry Inductively Coupled Plasma-Mass Spectrometry Inductively Coupled Plasma-Mass Spectrometry Inductively Coupled Plasma-Atomic Emission Spectrometry Inductively Coupled Plasma-Mass Spectrometry Inductively Coupled Plasma-Mass Spectrometry Inductively Coupled Plasma-Mass Spectrometry Inductively Coupled Plasma-Mass Spectrometry Inductively Coupled Plasma-Mass Spectrometry Inductively Coupled Plasma-Mass Spectrometry Inductively Coupled Plasma-Mass Spectrometry Inductively Coupled Plasma-Mass Spectrometry Inductively Coupled Plasma-Mass Spectrometry Inductively Coupled Plasma-Mass Spectrometry Inductively Coupled Plasma-Mass Spectrometry Inductively Coupled Plasma-Mass Spectrometry

\begin{tabular}{lll}
\hline & Suspended-sediment \\
\hline Suspended-sediment & Guy (1969) & Evaporation \\
Percent finer than $0.062 \mathrm{~mm}$ & Guy (1969) & Pipet \\
\hline
\end{tabular}


Table 4. Physical field parameters measured at surface-water sites in the Chulitna River basin, Alaska, March $2010-J u n e 2012$.

[Number in parentheses below constituent is number used by both U.S. Environmental Protection Agency and U.S. Geological Survey to identify parameters in computerized databases. Values represent the average of the cross section. Measurements were taken at four to five locations along the cross section. Site No.: Location of site No. is shown in figure 3. Abbreviations: $\mathrm{ft} / \mathrm{s}$, cubic foot per second; mg/L, milligram per liter; $\mu \mathrm{s} / \mathrm{cm}$ at $25^{\circ} \mathrm{C}$, microsiemens per centimeter at 25 degrees Celsius; ${ }^{\circ} \mathrm{C}$, degrees Celsius; LED, light-emitting diode; nm, nanometer; FNU, Formazin Nephelometric Unit; <, less than; -, not measured]

\begin{tabular}{|c|c|c|c|c|c|c|c|c|}
\hline $\begin{array}{l}\text { Site } \\
\text { No. }\end{array}$ & Date & Time & $\begin{array}{c}\text { Streamflow } \\
\left(\mathrm{ft}^{3} / \mathrm{s}\right) \\
(00061)\end{array}$ & $\begin{array}{c}\text { Dissolved } \\
\text { oxygen } \\
(\mathrm{mg} / \mathrm{L}) \\
(00300)\end{array}$ & $\begin{array}{c}\text { pH } \\
\text { (units) } \\
\text { (00400) }\end{array}$ & $\begin{array}{l}\text { Specific } \\
\text { conductance } \\
\text { ( } \mu \mathrm{s} / \mathrm{cm} \text { at } \\
\left.25^{\circ} \mathrm{C}\right) \\
(00095)\end{array}$ & $\begin{array}{c}\text { Water } \\
\text { temperature } \\
\left({ }^{\circ} \mathrm{C}\right) \\
(\mathbf{0 0 0 1 0})\end{array}$ & $\begin{array}{l}\text { Turbidity, water, unfiltered, } \\
\text { near infra-red LED light, } \\
780-900 \mathrm{~nm} \text { detection angle } \\
90 \pm 2.5 \text { degrees (FNU) } \\
\text { (63680) }\end{array}$ \\
\hline \multicolumn{9}{|c|}{ Chulitna River 0.4 mile above Nikabuna Lake, near Nondalton } \\
\hline \multirow[t]{3}{*}{1} & $03-25-2010$ & 1504 & 52 & 6.8 & 6.8 & 131 & 0.4 & - \\
\hline & 08-23-2011 & 1430 & 1,020 & 9.5 & 7.0 & 61 & 11.4 & 9.2 \\
\hline & 03-14-2012 & 1325 & 88 & 9.4 & 7.0 & 122 & 0.0 & $<2$ \\
\hline \multicolumn{9}{|c|}{ Hoknede Lake Creek 0.4 mile below Hoknede Lake, near Nondalton } \\
\hline 2 & 03-24-2010 & 1410 & 2.9 & 9.5 & 6.8 & 63 & 1.4 & - \\
\hline \multicolumn{9}{|c|}{ Koksetna River 0.1 mile above mouth, near Nondalton } \\
\hline \multirow[t]{5}{*}{3} & 03-25-2010 & 1215 & 109 & 13.0 & 7.4 & 97 & 0.0 & - \\
\hline & $07-22-2010$ & 1344 & 1,070 & 11.7 & 7.9 & 79 & 10.0 & 3.1 \\
\hline & 03-08-2011 & 1535 & 76 & 13.3 & 7.4 & 98 & 0.0 & $<2.0$ \\
\hline & 08-24-2011 & 1341 & 1,310 & 12.0 & 7.6 & 79 & 8.0 & $<2.0$ \\
\hline & 03-12-2012 & 1730 & 159 & 14 & 7.3 & 91 & 0.0 & $<2$ \\
\hline \multicolumn{9}{|c|}{ Chulitna River 0.3 mile above Koksetna River, near Nondalton } \\
\hline \multirow[t]{3}{*}{4} & 03-25-2010 & 1050 & 106 & 6.4 & 6.8 & 129 & 0.0 & - \\
\hline & 07-22-2010 & 1445 & 1,100 & 10.5 & 7.7 & 65 & 12.4 & 4.8 \\
\hline & 03-08-2011 & 1418 & 138 & 9.4 & 6.9 & 126 & 0.0 & $<2.0$ \\
\hline & 08-18-2010 & 1531 & 5,830 & 10.7 & 7.3 & 57 & 10.0 & 5.1 \\
\hline & 09-29-2010 & 1300 & 1,830 & 12.3 & 7.5 & 78 & 3.3 & 6.1 \\
\hline & 03-09-2011 & 1100 & 331 & 10.7 & 6.9 & 108 & 0.0 & $<2.0$ \\
\hline & 05-26-2011 & 1752 & 2,580 & 12.1 & 7.5 & 63 & 7.8 & 8.4 \\
\hline & 07-13-2011 & 1410 & 2,530 & 11.1 & 7.4 & 68 & 10.2 & 2.5 \\
\hline & 08-24-2011 & 1656 & 3,190 & 11.5 & 7.4 & 72 & 10.2 & $<2.0$ \\
\hline & 09-27-2011 & 1728 & 1,960 & 12.0 & 7.3 & 76 & 7.0 & $<2.0$ \\
\hline & 03-14-2012 & 1545 & 457 & 9.9 & 7.0 & 105 & 0.0 & $<2.0$ \\
\hline & 06-19-2012 & 1408 & 3,480 & 11.5 & 7.5 & 66 & 10.3 & 7.0 \\
\hline
\end{tabular}


Table 5. Concentrations of major ions in water samples collected at USGS streamflow-gaging station 15298040, Chulitna River 5 miles above mouth, near Port Alsworth, Alaska, March 2010-June 2012.

[Number in parentheses below constituent is number used by both U.S. Environmental Protection Agency and U.S. Geological Survey to identify parameters in computerized databases. Location of Site No. is shown in figure 3. All values in milligrams per liter. Abbreviation: E, estimated]

\begin{tabular}{|c|c|c|c|c|c|c|c|c|c|c|c|c|}
\hline Date & Time & $\begin{array}{c}\text { Alkalinity } \\
\text { (39086) }\end{array}$ & $\begin{array}{c}\text { Bicarbonate } \\
(00453)\end{array}$ & $\begin{array}{l}\text { Calcium } \\
\text { (00915) }\end{array}$ & $\begin{array}{c}\text { Chloride } \\
\text { (00940) }\end{array}$ & $\begin{array}{c}\text { Dissolved } \\
\text { solids } \\
(70300)\end{array}$ & $\begin{array}{c}\text { Fluoride } \\
\text { (00950) }\end{array}$ & $\begin{array}{c}\text { Magnesium } \\
\text { (00925) }\end{array}$ & $\begin{array}{l}\text { Potassium } \\
\text { (00935) }\end{array}$ & $\begin{array}{l}\text { Silica } \\
\text { (00955) }\end{array}$ & $\begin{array}{l}\text { Sodium } \\
\text { (00930) }\end{array}$ & $\begin{array}{l}\text { Sulfate } \\
\text { (00945) }\end{array}$ \\
\hline 03-23-2010 & 1810 & 46 & 56 & 14.5 & 0.808 & 66 & 0.080 & 2.48 & 0.694 & 12.0 & 2.66 & 8.43 \\
\hline 05-25-2010 & 1530 & 23 & 28 & 7.38 & .590 & 48 & .084 & 1.39 & .610 & 6.34 & 1.68 & 4.20 \\
\hline 07-21-2010 & 1720 & 33 & 40 & 9.10 & .348 & 61 & E.058 & 1.77 & .320 & 7.15 & 1.79 & 5.78 \\
\hline 09-29-2010 & 1230 & 28 & 34 & 10.2 & .497 & 51 & E.061 & 1.88 & .402 & 8.15 & 2.17 & 6.67 \\
\hline 03-09-2011 & 1740 & 40 & 49 & 15.3 & .709 & 66 & .062 & 2.65 & .632 & 11.8 & 2.72 & 8.80 \\
\hline 05-26-2011 & 1740 & 22 & 27 & 8.43 & .543 & 42 & .049 & 1.54 & .486 & 6.91 & 1.77 & 6.29 \\
\hline 07-13-2011 & 1320 & 25 & 31 & 8.97 & .380 & 50 & .061 & 1.73 & .318 & 8.03 & 1.86 & 5.36 \\
\hline 08-24-2011 & 1710 & 29 & 35 & 8.96 & .422 & 58 & .056 & 1.79 & .349 & 8.41 & 1.94 & 5.18 \\
\hline 09-27-2011 & 1740 & 30 & 37 & 10.2 & .614 & 60 & .041 & 1.96 & .454 & 9.13 & 2.02 & 5.08 \\
\hline
\end{tabular}


Table 6. Dissolved trace-element concentrations in water samples collected at USGS streamflow-gaging station 15298040, Chulitna River 5 miles above mouth, near Port Alsworth, Alaska, March 2010-June 2012.

[Number in parentheses below constituent is number used by both U.S. Environmental Protection Agency and U.S. Geological Survey to identify parameters in computerized databases. Location of Site No. is shown in figure 3. All values in micrograms per liter. Abbreviations: E,estimated; $<$, less than]

\begin{tabular}{|c|c|c|c|c|c|c|}
\hline \multicolumn{7}{|c|}{ Site 5 - Chulitna River 5 miles above mouth, near Port Alsworth (15298040) } \\
\hline Date & Time & $\begin{array}{c}\text { Aluminum } \\
\text { (01106) }\end{array}$ & $\begin{array}{c}\text { Antimony } \\
\text { (01095) }\end{array}$ & $\begin{array}{l}\text { Arsenic } \\
(01000)\end{array}$ & $\begin{array}{l}\text { Barium } \\
\text { (01005) }\end{array}$ & $\begin{array}{c}\text { Beryllium } \\
\text { (01010) }\end{array}$ \\
\hline 03-23-2010 & 1810 & E2.9 & 0.09 & 1.21 & 7.08 & $<0.012$ \\
\hline $05-25-2010$ & 1530 & 18.3 & .19 & 1.56 & 4.25 & $<.012$ \\
\hline 07-21-2010 & 1720 & 17.1 & .145 & 1.27 & 4.24 & $<.012$ \\
\hline $08-18-2010$ & 1450 & 45.0 & .099 & 1.21 & 3.79 & Е.006 \\
\hline 09-29-2010 & 1230 & 12.2 & .174 & 1.33 & 4.20 & $<.012$ \\
\hline 03-09-2011 & 1740 & 3.8 & .116 & 1.02 & 5.47 & $<.006$ \\
\hline 05-26-2011 & 1740 & 17.9 & .143 & .943 & 4.28 & $<.006$ \\
\hline 07-13-2011 & 1320 & 19.6 & .108 & 1.25 & 4.21 & $<.006$ \\
\hline 08-24-2011 & 1710 & 16.1 & .096 & 1.59 & 3.87 & $<.006$ \\
\hline 09-27-2011 & 1740 & 12.6 & .084 & 1.68 & 4.52 & $<.006$ \\
\hline 03-14-2012 & 1510 & 4.4 & .085 & 1.31 & 6.46 & $<.006$ \\
\hline 06-19-2012 & 1350 & 9.9 & .129 & 1.16 & 3.67 & $<.006$ \\
\hline $\begin{array}{l}\text { Boron } \\
(01020)\end{array}$ & $\begin{array}{l}\text { Cadmium } \\
\text { (01025) }\end{array}$ & $\begin{array}{l}\text { Chromium } \\
(01030)\end{array}$ & $\begin{array}{l}\text { Cobalt } \\
\text { (01035) }\end{array}$ & $\begin{array}{l}\text { Copper } \\
\text { (01040) }\end{array}$ & $\begin{array}{c}\text { Iron } \\
\text { (01046) }\end{array}$ & $\begin{array}{l}\text { Lead } \\
\text { (01049) }\end{array}$ \\
\hline 3.95 & $<0.02$ & E0.067 & 0.806 & $<1.0$ & 257 & 0.122 \\
\hline E2.72 & E.013 & E.104 & 1.040 & E.92 & 356 & .0699 \\
\hline 2.94 & $<.02$ & E.101 & .268 & E.87 & 151 & .0677 \\
\hline 2.98 & $<.02$ & .154 & .157 & E.70 & 200 & .0449 \\
\hline E2.73 & $<.02$ & .121 & 1.690 & E.64 & 263 & .0504 \\
\hline 3.68 & $<.016$ & .090 & .534 & $<.50$ & 212 & .0239 \\
\hline$<3$ & $<.016$ & .072 & .798 & .51 & 176 & .0716 \\
\hline$<3$ & $<.016$ & .137 & .200 & .55 & 162 & .0595 \\
\hline$<3$ & $<.016$ & .116 & .334 & .56 & 241 & .0380 \\
\hline$<3$ & $<.016$ & .099 & 1.480 & $<.50$ & 363 & .0432 \\
\hline 3.83 & $<.016$ & .303 & .072 & $<.80$ & 310 & .0251 \\
\hline 3.40 & $<.016$ & .076 & .402 & $<.80$ & 125 & .1315 \\
\hline $\begin{array}{l}\text { Lithium } \\
\text { (01130) }\end{array}$ & $\begin{array}{l}\text { Manganese } \\
\text { (01056) }\end{array}$ & $\begin{array}{l}\text { Molybdenum } \\
(01060)\end{array}$ & $\begin{array}{l}\text { Nickel } \\
\text { (01065) }\end{array}$ & $\begin{array}{l}\text { Selenium } \\
\text { (01145) }\end{array}$ & $\begin{array}{c}\text { Silver } \\
\text { (01075) }\end{array}$ & $\begin{array}{c}\text { Strontium } \\
\text { (01080) }\end{array}$ \\
\hline 1.30 & 36.7 & 0.375 & 0.239 & 0.152 & $<0.010$ & 76.4 \\
\hline .571 & 14.2 & .304 & .471 & .0688 & $<.010$ & 39.4 \\
\hline .577 & 15.3 & .280 & .266 & .0800 & $<.010$ & 52.4 \\
\hline .656 & 11.0 & .277 & .302 & .0947 & E.006 & 43.2 \\
\hline .600 & 26.2 & .316 & .641 & .0898 & $<.010$ & 56.2 \\
\hline .942 & 30.2 & .343 & .509 & .1454 & $<.005$ & 72.5 \\
\hline .573 & 10.0 & .319 & .497 & .1132 & $<.005$ & 44.9 \\
\hline .665 & 12.7 & .356 & .241 & .1173 & $<.005$ & 50.0 \\
\hline .690 & 12.7 & .341 & .337 & .1017 & $<.005$ & 57.1 \\
\hline .841 & 22.7 & .357 & .402 & .0897 & $<.005$ & 57.4 \\
\hline .944 & 62.1 & .394 & .202 & .1211 & $<.005$ & 78.5 \\
\hline .592 & 10.3 & .353 & .242 & .1207 & $<.005$ & 47.4 \\
\hline $\begin{array}{l}\text { Thallium } \\
\text { (01057) }\end{array}$ & $\begin{array}{l}\text { Uranium } \\
(22703)\end{array}$ & $\begin{array}{l}\text { Vanadium } \\
\text { (01085) }\end{array}$ & $\begin{array}{c}\text { Zinc } \\
(01090)\end{array}$ & & & \\
\hline$<0.020$ & 0.0293 & E0.139 & $<2.8$ & & & \\
\hline$<.020$ & .0221 & .521 & $<2.8$ & & & \\
\hline$<.020$ & .0234 & .314 & $<2.8$ & & & \\
\hline$<.020$ & .0266 & .360 & $<2.8$ & & & \\
\hline$<.020$ & .0257 & .238 & $<2.8$ & & & \\
\hline$<.010$ & .0233 & .156 & $<1.4$ & & & \\
\hline$<.010$ & .0170 & .307 & $<1.4$ & & & \\
\hline$<.010$ & .0223 & .266 & $<1.4$ & & & \\
\hline$<.010$ & .0235 & .272 & $<1.4$ & & & \\
\hline$<.010$ & .0257 & .279 & $<1.4$ & & & \\
\hline .066 & .0287 & .162 & $<1.4$ & & & \\
\hline$<.010$ & .0216 & .220 & $<1.4$ & & & \\
\hline
\end{tabular}


Table 7. Concentrations of nutrients, dissolved organic carbon, and suspended sediment in water samples collected at USGS streamflow-gaging station 15298040, Chulitna River 5 miles above mouth, near Port Alsworth, Alaska, March $2010-J u n e ~ 2012$.

[Number in parentheses below constituent is number used by both U.S. Environmental Protection Agency and U.S. Geological Survey to identify parameters in computerized databases. All values in milligrams per liter unless otherwise noted. Abbreviations: mm, millimeter; E, estimated; <, less than; -, constituent not measured]

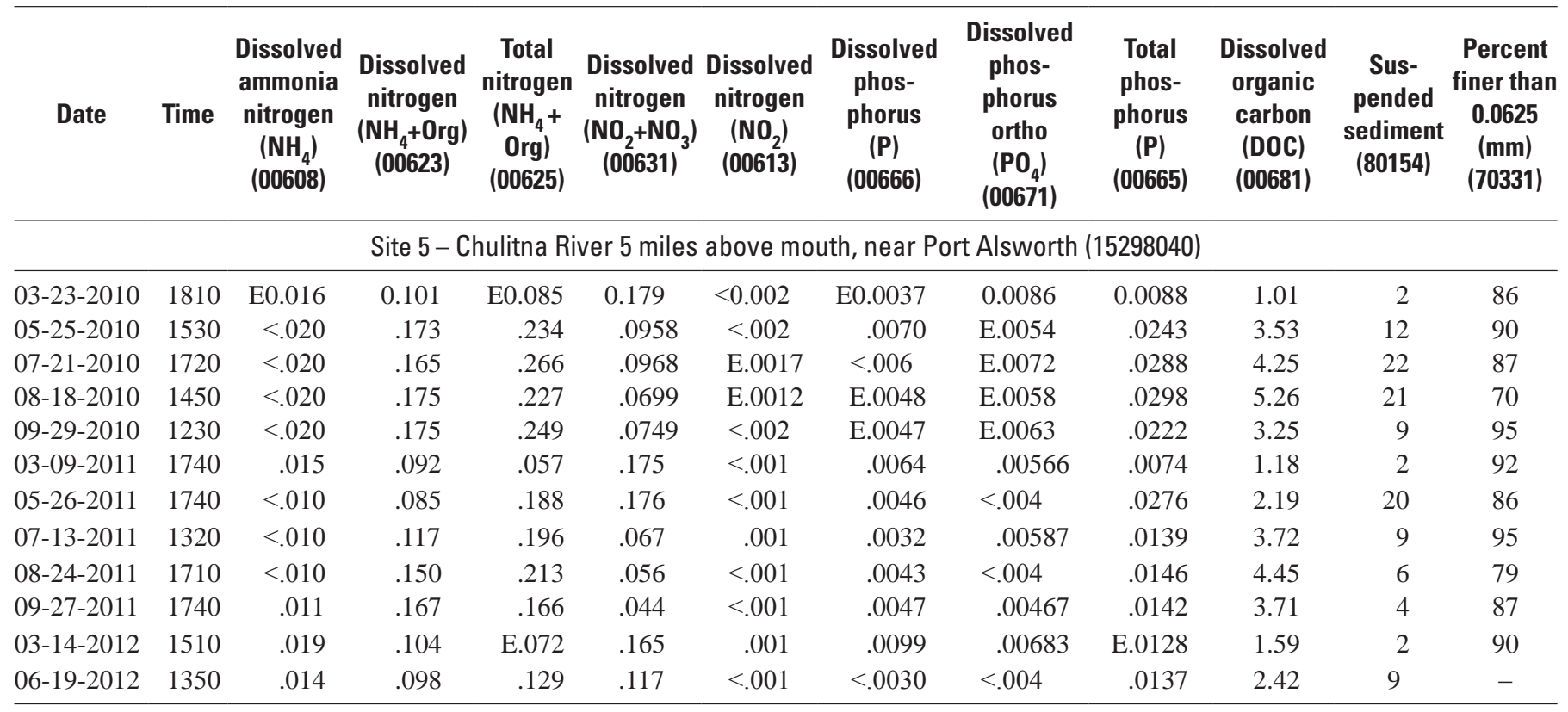

Table 8. Results from a blank sample collected at USGS streamflow-gaging station 15298040, Chulitna River 5 miles above mouth, near Port Alsworth, Alaska, August 18, 2010.

[Number in parentheses below constituent is number used by both U.S. Environmental Protection Agency and U.S. Geological Survey to identify parameters in computerized databases. All values in milligrams per liter unless otherwise noted. Abbreviations: $\mu \mathrm{g} / \mathrm{L}$, microgram per liter; E, estimated; <, less than]

\begin{tabular}{|c|c|c|c|c|c|c|c|c|c|c|c|}
\hline Date & Time & $\begin{array}{c}\text { Alkalinity } \\
\text { (39086) }\end{array}$ & $\begin{array}{c}\text { Calcium } \\
\text { (00915) }\end{array}$ & $\begin{array}{l}\text { Chloride } \\
\text { (00940) }\end{array}$ & $\begin{array}{l}\text { Dissolved } \\
\text { solids } \\
(70300)\end{array}$ & $\begin{array}{c}\text { Fluoride } \\
\text { (00950) }\end{array}$ & $\begin{array}{c}\text { Magnesium } \\
\text { (00925) }\end{array}$ & $\begin{array}{l}\text { Potassium } \\
\text { (00935) }\end{array}$ & $\begin{array}{l}\text { Silica } \\
\text { (00955) }\end{array}$ & $\begin{array}{l}\text { Sodium } \\
\text { (00930) }\end{array}$ & $\begin{array}{l}\text { Sulfate } \\
\text { (00945) }\end{array}$ \\
\hline $\begin{array}{c}\text { Dissolved } \\
\text { ammonia } \\
\text { nitrogen } \\
\left(\mathrm{NH}_{4}\right) \\
(00608)\end{array}$ & $\begin{array}{c}\text { Dissolved } \\
\text { nitrogen } \\
\left(\mathrm{NH}_{4}+\mathrm{Org}\right) \\
(00623)\end{array}$ & $\begin{array}{c}\text { Total } \\
\text { nitrogen } \\
\left(\mathrm{NH}_{4}+\text { Org) }\right. \\
(00625)\end{array}$ & $\begin{array}{c}\text { Dissolved } \\
\text { nitrogen } \\
\left(\mathrm{NO}_{2}+\mathrm{NO}_{3}\right) \\
(00631)\end{array}$ & $\begin{array}{c}\text { Dissolved } \\
\text { nitrogen } \\
\left(\mathrm{NO}_{2}\right) \\
(00613)\end{array}$ & $\begin{array}{c}\text { Dissolved } \\
\text { phosphorus } \\
\text { (P) } \\
(00666)\end{array}$ & $\begin{array}{c}\text { Phosphorus } \\
\text { ortho } \\
\left(\mathrm{PO}_{4}\right) \\
(00671)\end{array}$ & $\begin{array}{c}\text { Total } \\
\text { phosphorus } \\
\text { (P) } \\
(00665)\end{array}$ & $\begin{array}{c}\text { Dissolved } \\
\text { organic } \\
\text { carbon } \\
\text { (DOC) } \\
\text { (00681) }\end{array}$ & $\begin{array}{c}\text { Aluminum } \\
(01106) \\
(\mu \mathrm{g} / \mathrm{L})\end{array}$ & $\begin{array}{c}\text { Antimony } \\
\text { (01095) } \\
(\mu \mathrm{g} / \mathrm{L})\end{array}$ & $\begin{array}{c}\text { Arsenic } \\
(01000) \\
(\mu \mathrm{g} / \mathrm{L})\end{array}$ \\
\hline$<0.020$ & $<0.10$ & $<0.10$ & $<0.016$ & $<0.002$ & $<0.006$ & $<0.008$ & $<0.008$ & $<0.66$ & $<3.4$ & E0.04 & $<0.04$ \\
\hline $\begin{array}{c}\text { Barium } \\
\text { (01005) } \\
(\mu \mathrm{g} / \mathrm{L})\end{array}$ & $\begin{array}{c}\text { Beryllium } \\
\text { (01010) } \\
(\mu \mathrm{g} / \mathrm{L})\end{array}$ & $\begin{array}{c}\text { Boron } \\
(01020) \\
(\mu \mathrm{g} / \mathrm{L})\end{array}$ & $\begin{array}{c}\text { Cadmium } \\
\text { (01025) } \\
(\mu \mathrm{g} / \mathrm{L})\end{array}$ & $\begin{array}{c}\text { Chromium } \\
(01080) \\
(\mu \mathrm{g} / \mathrm{L})\end{array}$ & $\begin{array}{c}\text { Cobalt } \\
\text { (01035) } \\
(\mu \mathrm{g} / \mathrm{L})\end{array}$ & $\begin{array}{c}\text { Copper } \\
\text { (01040) } \\
(\mu g / L)\end{array}$ & $\begin{array}{c}\text { Iron } \\
(10146) \\
(\mu \mathrm{g} / \mathrm{L})\end{array}$ & $\begin{array}{c}\text { Lead } \\
(01049) \\
(\mu \mathrm{g} / \mathrm{L})\end{array}$ & $\begin{array}{c}\text { Lithium } \\
\text { (01130) } \\
\text { ( } \mu \mathrm{g} / \mathrm{L})\end{array}$ & $\begin{array}{c}\text { Manganese } \\
(01056) \\
(\mu \mathrm{g} / \mathrm{L})\end{array}$ & $\begin{array}{c}\text { Molyb- } \\
\text { denum } \\
(01060) \\
(\mu \mathrm{g} / \mathrm{L})\end{array}$ \\
\hline $\begin{array}{c}\text { Nickel } \\
\text { (01065) } \\
\text { ( } \mu \mathrm{g} / \mathrm{L})\end{array}$ & $\begin{array}{c}\text { Selenium } \\
(01145) \\
(\mu \mathrm{g} / \mathrm{L})\end{array}$ & $\begin{array}{c}\text { Silver } \\
\text { (01075) } \\
(\mu \mathrm{g} / \mathrm{L})\end{array}$ & $\begin{array}{c}\text { Strontium } \\
(01080) \\
(\mu \mathrm{g} / \mathrm{L})\end{array}$ & $\begin{array}{c}\text { Thallium } \\
\text { (01057) } \\
(\mu \mathrm{g} / \mathrm{L})\end{array}$ & $\begin{array}{c}\text { Uranium } \\
(22703) \\
(\mu \mathrm{g} / \mathrm{L})\end{array}$ & $\begin{array}{c}\text { Vanadium } \\
\text { (01085) } \\
(\mu \mathrm{g} / \mathrm{L})\end{array}$ & $\begin{array}{c}\text { Zinc } \\
\text { (01090) } \\
(\mu \mathrm{g} / \mathrm{L})\end{array}$ & & & & \\
\hline$<0.12$ & $<0.04$ & $<0.010$ & $<0.40$ & $<0.020$ & $<0.008$ & $<0.16$ & $<2.8$ & & & & \\
\hline
\end{tabular}


Table 9. Results from a replicate sample collected at USGS streamflow-gaging station 15298040, Chulitna River 5 miles above mouth, near Port Alsworth, Alaska, March 9, 2011.

[Number in parentheses below constituent is number used by both U.S. Environmental Protection Agency and U.S. Geological Survey to identify parameters in computerized databases. Abbreviations: mg/L, milligram per liter; $\mu \mathrm{g} / \mathrm{L}$, microgram per liter; E, estimated; <, less than]

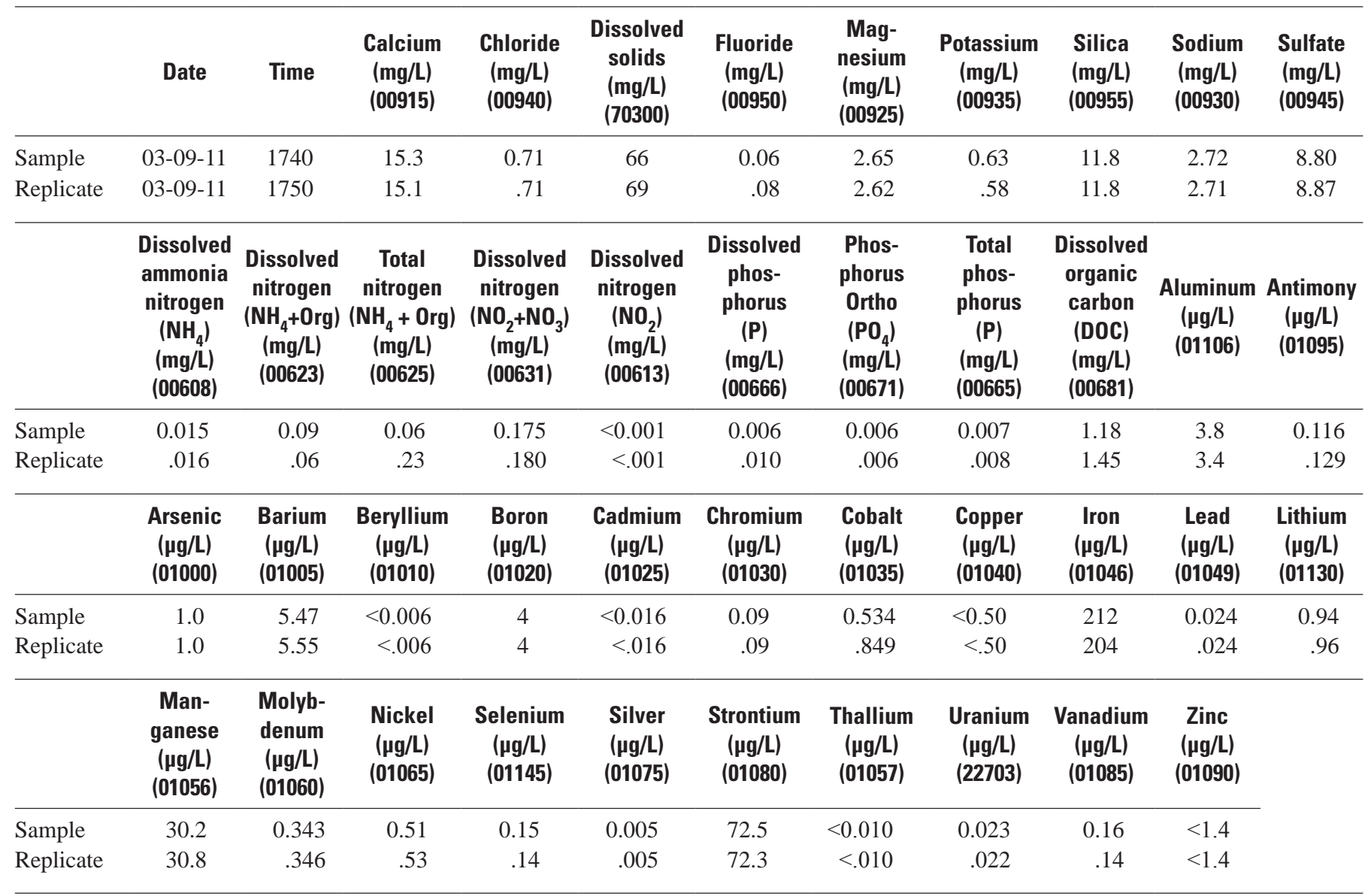


Table 10. Water temperature data for USGS streamflow-gaging station 15298040, Chulitna River 5 miles above mouth, near Port Alsworth, Alaska, water years 2010 and 2011.

[Abbreviations: Max, maximum; Min, minimum; -, no data]

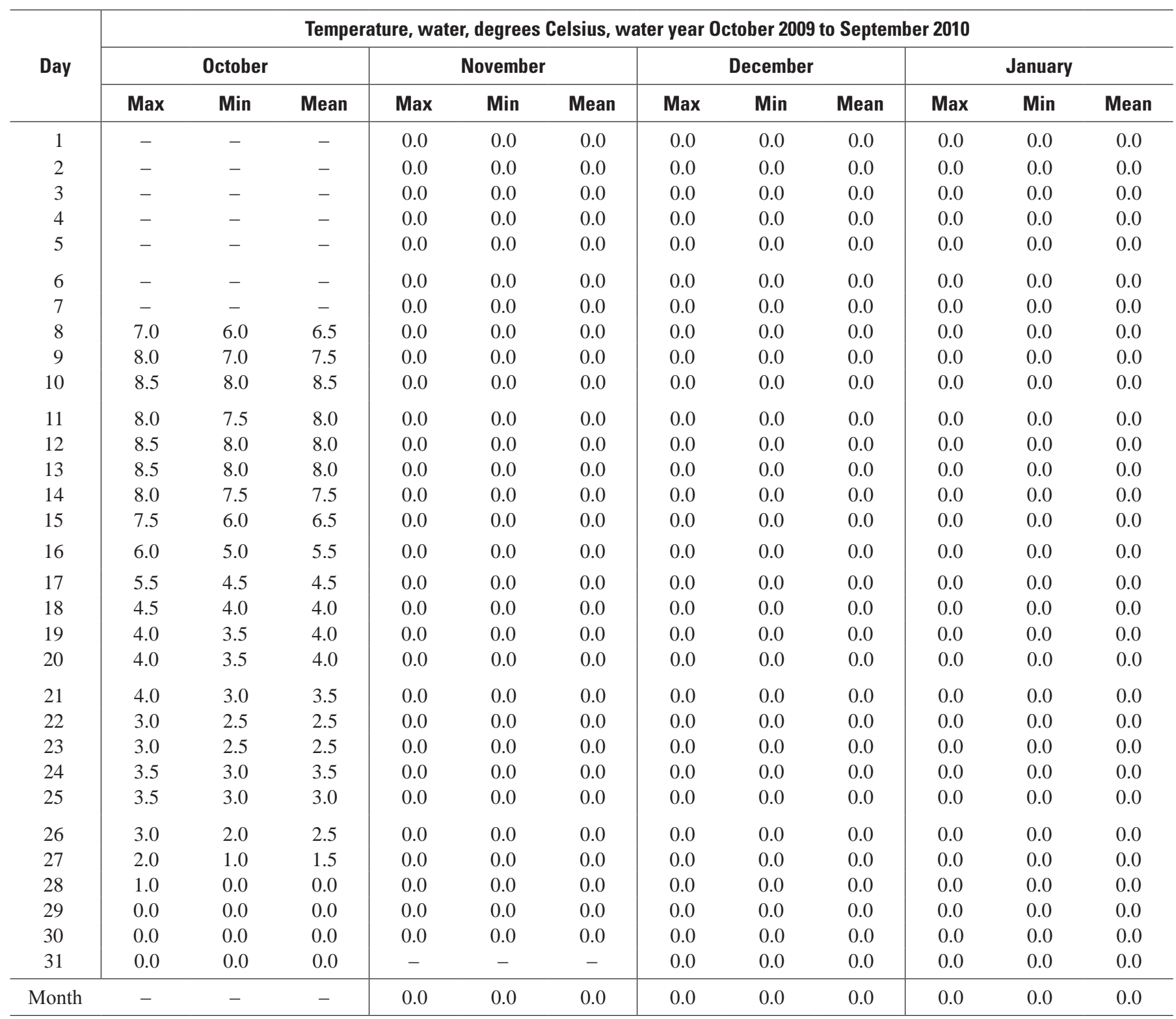


Table 10. Water temperature data for USGS streamflow-gaging station 15298040, Chulitna River 5 miles above mouth, near Port Alsworth, Alaska, water years 2010 and 2011.-Continued

[Abbreviations: Max, maximum; Min, minimum; -, no data]

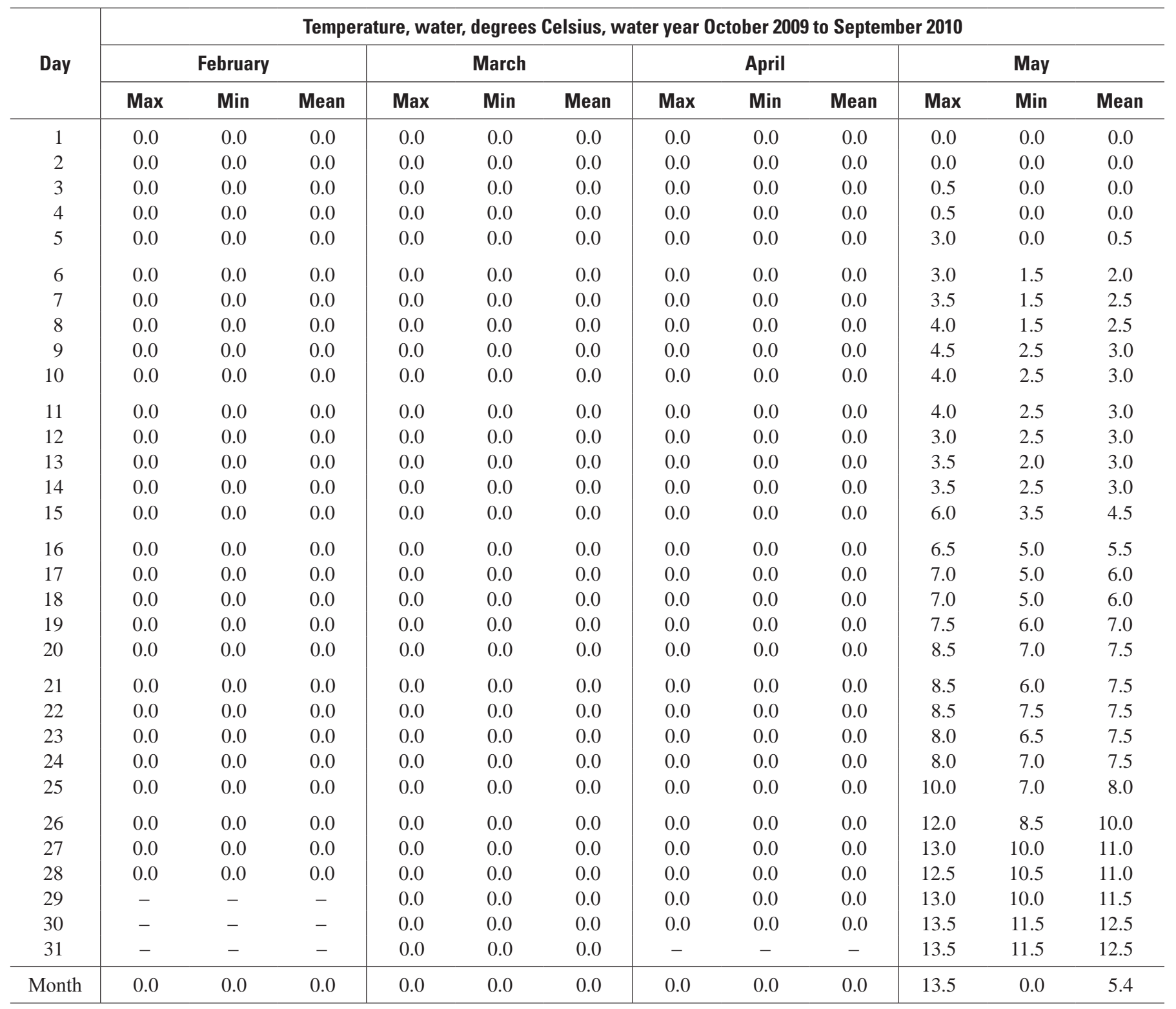


Table 10. Water temperature data for USGS streamflow-gaging station 15298040, Chulitna River 5 miles above mouth, near Port Alsworth, Alaska, water years 2010 and 2011. - Continued

[Abbreviations: Max, maximum; Min, minimum; -, no data]

\begin{tabular}{|c|c|c|c|c|c|c|c|c|c|c|c|c|}
\hline \multirow{3}{*}{ Day } & \multicolumn{12}{|c|}{ Temperature, water, degrees Celsius, water year October 2009 to September 2010} \\
\hline & \multicolumn{3}{|c|}{ June } & \multicolumn{3}{|c|}{ July } & \multicolumn{3}{|c|}{ August } & \multicolumn{3}{|c|}{ September } \\
\hline & Max & Min & Mean & Max & Min & Mean & Max & Min & Mean & Max & Min & Mean \\
\hline 1 & 14.0 & 11.5 & 12.5 & 14.5 & 12.5 & 13.0 & 11.0 & 10.0 & 10.5 & 11.5 & 10.5 & 11.0 \\
\hline 2 & 13.5 & 12.0 & 12.5 & 12.5 & 11.0 & 12.0 & 13.5 & 10.5 & 11.5 & 11.0 & 10.0 & 10.5 \\
\hline 3 & 12.5 & 10.5 & 11.5 & 13.0 & 11.5 & 12.0 & 13.5 & 12.0 & 12.5 & 11.0 & 9.5 & 10.5 \\
\hline 4 & 10.5 & 9.5 & 10.0 & 13.0 & 12.0 & 12.5 & 12.5 & 12.0 & 12.0 & 11.0 & 10.5 & 10.5 \\
\hline 5 & 10.0 & 7.5 & 9.0 & 12.5 & 11.5 & 12.0 & 12.5 & 11.5 & 12.0 & 11.0 & 10.0 & 10.5 \\
\hline 6 & 11.0 & 9.0 & 10.0 & 11.5 & 10.5 & 10.5 & 12.0 & 11.0 & 11.5 & 11.0 & 10.0 & 10.5 \\
\hline 7 & 11.5 & 10.0 & 10.5 & 10.5 & 10.0 & 10.0 & 11.0 & 10.0 & 10.5 & 11.0 & 10.5 & 11.0 \\
\hline 8 & 12.5 & 10.0 & 11.5 & 12.5 & 9.5 & 11.0 & 10.0 & 10.0 & 10.0 & 10.5 & 10.0 & 10.5 \\
\hline 9 & 12.5 & 10.5 & 11.0 & 12.5 & 11.0 & 12.0 & 10.0 & 10.0 & 10.0 & 10.0 & 9.5 & 10.0 \\
\hline 10 & 11.5 & 10.0 & 10.5 & 12.0 & 11.5 & 12.0 & 10.0 & 9.5 & 10.0 & 10.5 & 9.0 & 9.5 \\
\hline 11 & 12.5 & 9.5 & 11.0 & 12.0 & 11.0 & 11.5 & 10.0 & 9.0 & 9.5 & 10.5 & 9.0 & 9.5 \\
\hline 12 & 13.0 & 11.0 & 12.0 & 14.5 & 11.5 & 12.5 & 9.0 & 8.5 & 9.0 & 10.5 & 9.0 & 10.0 \\
\hline 13 & 14.0 & 11.5 & 12.5 & 14.5 & 13.0 & 13.5 & 10.0 & 9.0 & 9.5 & 11.0 & 9.5 & 10.0 \\
\hline 14 & 13.5 & 11.5 & 12.5 & 14.0 & 12.5 & 13.0 & 12.0 & 10.0 & 10.5 & 11.0 & 9.5 & 10.0 \\
\hline 15 & 11.5 & 9.5 & 10.5 & 14.0 & 12.5 & 13.0 & 12.0 & 11.5 & 11.5 & 11.0 & 9.0 & 10.0 \\
\hline 16 & 10.0 & 9.0 & 9.5 & 15.0 & 12.0 & 13.5 & 11.5 & 10.5 & 10.5 & 11.0 & 9.5 & 10.0 \\
\hline 17 & 10.5 & 9.0 & 9.5 & 16.0 & 13.5 & 14.5 & 10.5 & 10.0 & 10.5 & 10.5 & 9.5 & 10.0 \\
\hline 18 & 11.0 & 9.5 & 10.0 & 16.0 & 14.5 & 15.0 & 10.0 & 9.5 & 10.0 & 10.0 & 9.0 & 9.5 \\
\hline 19 & 12.5 & 10.0 & 11.0 & 14.5 & 12.5 & 13.5 & 10.5 & 9.0 & 9.5 & 10.0 & 8.5 & 9.5 \\
\hline 20 & 13.5 & 10.5 & 12.0 & 12.5 & 11.0 & 12.0 & 11.0 & 9.5 & 10.0 & 10.0 & 8.5 & 9.0 \\
\hline 21 & 15.0 & 12.0 & 13.0 & 11.5 & 10.0 & 11.0 & 11.0 & 10.0 & 10.5 & 9.5 & 8.5 & 9.0 \\
\hline 22 & 15.5 & 13.5 & 14.5 & 12.5 & 10.5 & 11.5 & 12.0 & 10.5 & 11.0 & 9.5 & 8.5 & 9.0 \\
\hline 23 & 17.0 & 14.5 & 15.5 & 12.0 & 10.0 & 11.0 & 12.5 & 10.5 & 11.5 & 8.5 & 7.5 & 8.0 \\
\hline 24 & 16.5 & 14.5 & 15.5 & 10.0 & 9.0 & 9.5 & 13.5 & 11.5 & 12.5 & 8.0 & 6.5 & 7.0 \\
\hline 25 & 15.5 & 13.5 & 14.5 & 9.0 & 9.0 & 9.0 & 13.5 & 12.0 & 12.5 & 7.0 & 5.5 & 6.0 \\
\hline 26 & 15.0 & 13.5 & 14.5 & 9.5 & 8.5 & 9.0 & 13.5 & 12.5 & 13.0 & 5.5 & 4.0 & 4.5 \\
\hline 27 & 15.0 & 13.5 & 14.0 & 10.0 & 9.0 & 9.5 & 13.0 & 12.5 & 12.5 & 4.0 & 2.5 & 3.0 \\
\hline 28 & 15.0 & 12.5 & 13.5 & 10.0 & 9.5 & 9.5 & 12.5 & 11.5 & 11.5 & 3.0 & 2.0 & 2.5 \\
\hline 29 & 14.5 & 13.5 & 14.0 & 10.0 & 9.0 & 9.5 & 12.0 & 10.5 & 11.5 & 4.0 & 2.5 & 3.5 \\
\hline 30 & 15.0 & 12.5 & 13.5 & 9.5 & 9.0 & 9.0 & 12.0 & 11.0 & 11.5 & 5.0 & 4.0 & 4.5 \\
\hline 31 & - & - & - & 10.0 & 9.5 & 9.5 & 12.0 & 11.0 & 11.5 & - & - & - \\
\hline Month & 17.0 & 7.5 & 12.1 & 16.0 & 8.5 & 11.5 & 13.5 & 8.5 & 11.0 & 11.5 & 2.0 & 8.6 \\
\hline
\end{tabular}


Table 10. Water temperature data for USGS streamflow-gaging station 15298040, Chulitna River 5 miles above mouth, near Port Alsworth, Alaska, water years 2010 and 2011.-Continued

[Abbreviations: Max, maximum; Min, minimum; -, no data]

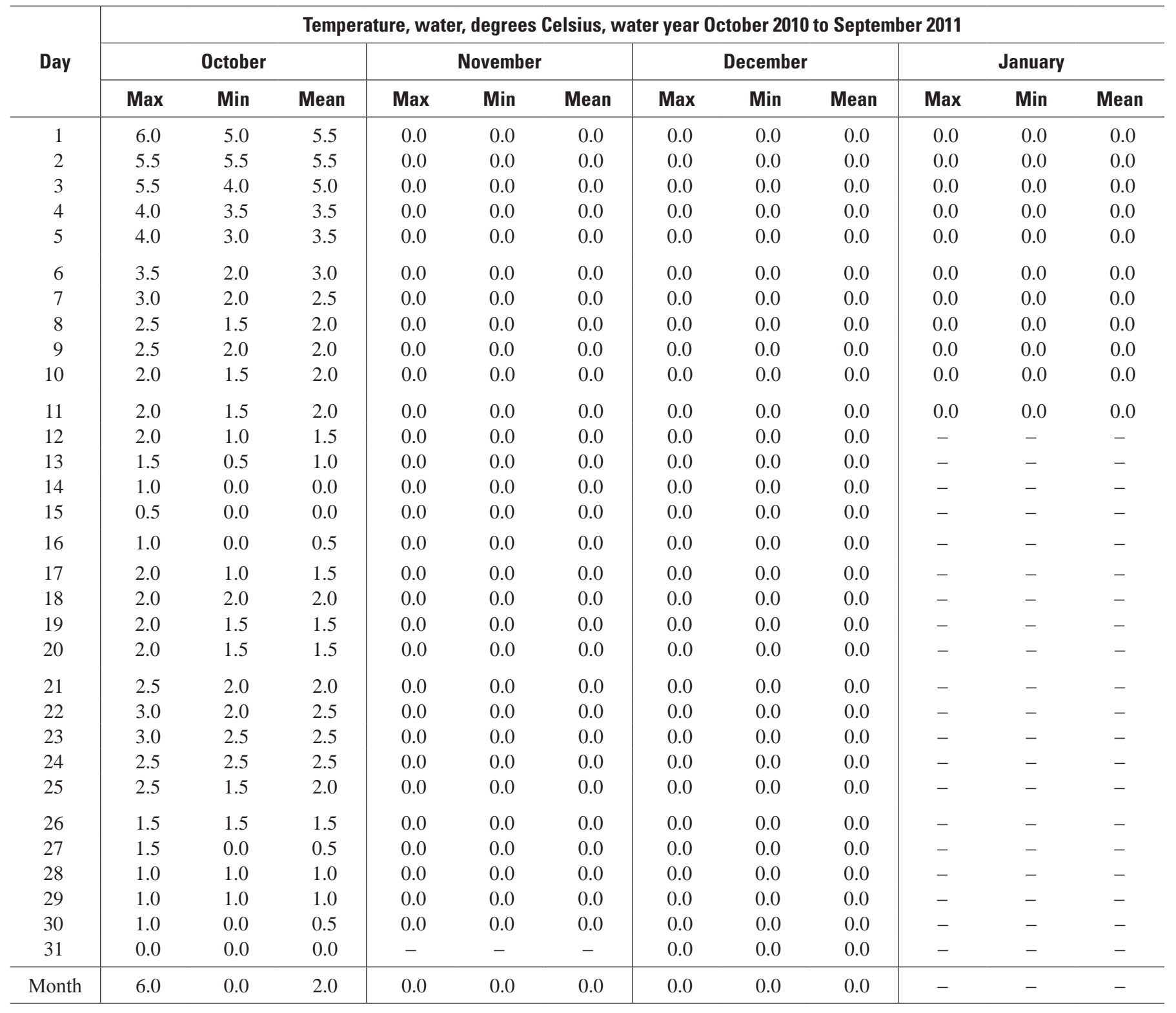


Table 10. Water temperature data for USGS streamflow-gaging station 15298040, Chulitna River 5 miles above mouth, near Port Alsworth, Alaska, water years 2010 and 2011.-Continued

[Abbreviations: Max, maximum; Min, minimum; -, no data]

\begin{tabular}{|c|c|c|c|c|c|c|c|c|c|c|c|c|}
\hline \multirow{3}{*}{ Day } & \multicolumn{12}{|c|}{ Temperature, water, degrees Celsius, water year October 2010 to September 2011} \\
\hline & \multicolumn{3}{|c|}{ February } & \multicolumn{3}{|c|}{ March } & \multicolumn{3}{|c|}{ April } & \multicolumn{3}{|c|}{ May } \\
\hline & Max & Min & Mean & Max & Min & Mean & Max & Min & Mean & Max & Min & Mean \\
\hline 1 & - & - & - & - & - & - & 0.0 & 0.0 & 0.0 & 0.0 & 0.0 & 0.0 \\
\hline 2 & - & - & - & - & - & - & 0.0 & 0.0 & 0.0 & 2.0 & 0.0 & 0.5 \\
\hline 3 & - & - & - & - & - & - & 0.0 & 0.0 & 0.0 & 3.0 & 1.0 & 2.0 \\
\hline 4 & - & - & - & - & - & - & 0.0 & 0.0 & 0.0 & 3.5 & 1.5 & 2.5 \\
\hline 5 & - & - & - & - & - & - & 0.0 & 0.0 & 0.0 & 3.5 & 1.5 & 2.5 \\
\hline 6 & - & - & - & - & - & - & 0.0 & 0.0 & 0.0 & 4.5 & 1.5 & 3.0 \\
\hline 7 & - & - & - & - & - & - & 0.0 & 0.0 & 0.0 & 4.0 & 2.0 & 3.0 \\
\hline 8 & - & - & - & - & - & - & 0.0 & 0.0 & 0.0 & 3.5 & 2.5 & 3.0 \\
\hline 9 & - & - & - & 0.0 & 0.0 & 0.0 & 0.0 & 0.0 & 0.0 & 4.0 & 2.5 & 3.0 \\
\hline 10 & - & - & - & 0.0 & 0.0 & 0.0 & 0.0 & 0.0 & 0.0 & 5.5 & 2.5 & 3.5 \\
\hline 11 & - & - & - & 0.0 & 0.0 & 0.0 & 0.0 & 0.0 & 0.0 & 6.0 & 3.5 & 4.5 \\
\hline 12 & - & - & - & 0.0 & 0.0 & 0.0 & 0.0 & 0.0 & 0.0 & 6.0 & 3.0 & 4.5 \\
\hline 13 & - & - & - & 0.0 & 0.0 & 0.0 & 0.0 & 0.0 & 0.0 & 7.5 & 4.0 & 5.5 \\
\hline 14 & - & - & - & 0.0 & 0.0 & 0.0 & 0.0 & 0.0 & 0.0 & 7.0 & 6.0 & 6.0 \\
\hline 15 & - & - & - & 0.0 & 0.0 & 0.0 & 0.0 & 0.0 & 0.0 & 6.0 & 5.0 & 5.5 \\
\hline 16 & - & - & - & 0.0 & 0.0 & 0.0 & 0.0 & 0.0 & 0.0 & 6.0 & 5.0 & 5.5 \\
\hline 17 & - & - & - & 0.0 & 0.0 & 0.0 & 0.0 & 0.0 & 0.0 & 6.0 & 5.0 & 5.5 \\
\hline 18 & - & - & - & 0.0 & 0.0 & 0.0 & 0.0 & 0.0 & 0.0 & 7.0 & 5.0 & 6.0 \\
\hline 19 & - & - & - & 0.0 & 0.0 & 0.0 & 0.0 & 0.0 & 0.0 & 7.0 & 6.5 & 7.0 \\
\hline 20 & - & - & - & 0.0 & 0.0 & 0.0 & 0.0 & 0.0 & 0.0 & 7.0 & 5.5 & 6.0 \\
\hline 21 & - & - & - & 0.0 & 0.0 & 0.0 & 0.0 & 0.0 & 0.0 & 8.0 & 6.0 & 7.0 \\
\hline 22 & - & - & - & 0.0 & 0.0 & 0.0 & 0.0 & 0.0 & 0.0 & 8.5 & 6.5 & 7.5 \\
\hline 23 & - & - & - & 0.0 & 0.0 & 0.0 & 0.0 & 0.0 & 0.0 & 8.5 & 7.0 & 7.5 \\
\hline 24 & - & - & - & 0.0 & 0.0 & 0.0 & 0.0 & 0.0 & 0.0 & 7.5 & 6.5 & 7.0 \\
\hline 25 & - & - & - & 0.0 & 0.0 & 0.0 & 0.0 & 0.0 & 0.0 & 8.5 & 5.5 & 7.0 \\
\hline 26 & - & - & - & 0.0 & 0.0 & 0.0 & 0.0 & 0.0 & 0.0 & 8.5 & 7.5 & 8.0 \\
\hline 27 & - & - & - & 0.0 & 0.0 & 0.0 & 0.0 & 0.0 & 0.0 & 10.0 & 7.5 & 8.5 \\
\hline 28 & - & - & - & 0.0 & 0.0 & 0.0 & 0.0 & 0.0 & 0.0 & 10.0 & 9.0 & 9.5 \\
\hline 29 & - & - & - & 0.0 & 0.0 & 0.0 & 0.0 & 0.0 & 0.0 & 9.5 & 8.5 & 9.0 \\
\hline 30 & - & - & - & 0.0 & 0.0 & 0.0 & 0.0 & 0.0 & 0.0 & 11.0 & 8.5 & 9.5 \\
\hline 31 & - & - & - & 0.0 & 0.0 & 0.0 & - & - & - & 11.0 & 9.5 & 10.0 \\
\hline Month & - & - & - & - & - & - & 0.0 & 0.0 & 0.0 & 11.0 & 0.0 & 5.5 \\
\hline
\end{tabular}


Table 10. Water temperature data for USGS streamflow-gaging station 15298040, Chulitna River 5 miles above mouth, near Port Alsworth, Alaska, water years 2010 and 2011._Continued

[Abbreviations: Max, maximum; Min, minimum; -, no data]

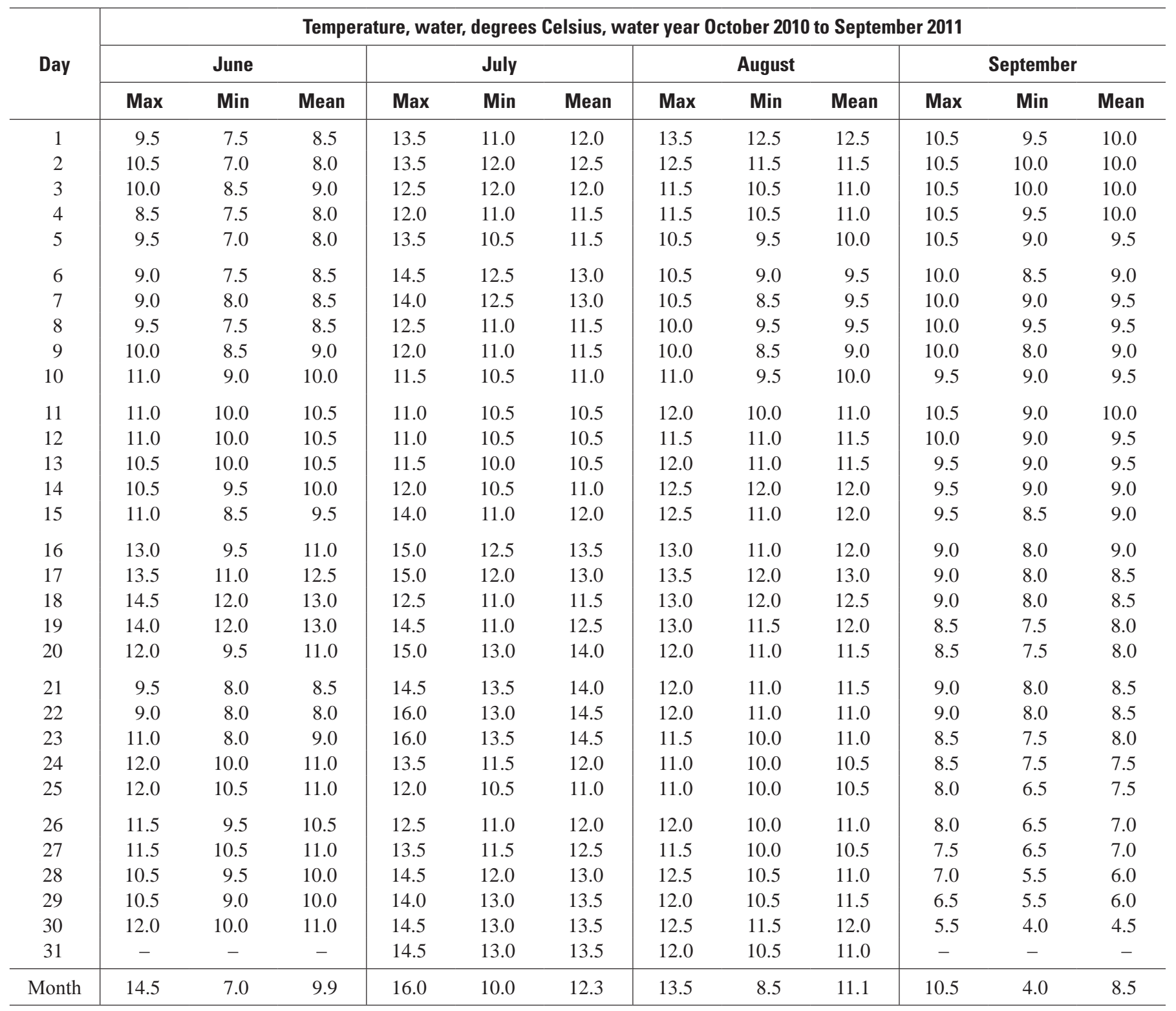


Table 11. Dissolved oxygen data for USGS streamflow-gaging station 15298040, Chulitna River 5 miles above mouth, near Port Alsworth, Alaska, water years 2010 and 2011.

[Abbreviations: Max, maximum; Min, minimum; -, no data]

\begin{tabular}{|c|c|c|c|c|c|c|c|c|c|c|c|c|}
\hline \multirow{2}{*}{ Day } & \multicolumn{12}{|c|}{ Dissolved oxygen, water, unfiltered, milligrams per liter, water year October 2009 to September 2010} \\
\hline & Max & Min & Max & Min & Max & Min & Max & Min & Max & Min & Max & Min \\
\hline 1 & - & - & - & - & 11.5 & 10.9 & 11.3 & 10.3 & 11.1 & 10.9 & 10.6 & 10.2 \\
\hline 2 & - & - & - & - & 11.4 & 10.7 & 11.4 & 10.8 & 11.0 & 10.6 & 10.7 & 10.3 \\
\hline 3 & - & - & - & - & 11.6 & 10.8 & 11.4 & 10.8 & 10.6 & 10.3 & 10.7 & 10.4 \\
\hline 6 & - & - & - & - & 12.4 & 11.8 & 11.8 & 11.0 & 10.4 & 10.1 & 10.5 & 10.3 \\
\hline 7 & - & - & - & - & 12.1 & 11.5 & 11.8 & 11.3 & 10.7 & 10.3 & 10.4 & 10.1 \\
\hline 8 & - & - & - & - & 12.0 & 11.5 & 11.9 & 11.3 & 10.7 & 10.6 & 10.7 & 10.3 \\
\hline 9 & - & - & - & - & 11.9 & 11.2 & 11.5 & 10.9 & 10.7 & 10.5 & 10.8 & 10.5 \\
\hline 10 & - & - & - & - & 12.1 & 11.4 & 11.3 & 10.6 & 10.8 & 10.5 & 10.9 & 10.5 \\
\hline 14 & - & - & - & - & 11.4 & 10.7 & 11.1 & 10.2 & 10.8 & 10.4 & 10.9 & 10.3 \\
\hline 15 & - & - & - & - & 11.8 & 11.0 & 11.1 & 10.3 & 10.4 & 10.1 & 10.9 & 10.4 \\
\hline 16 & - & - & - & - & 12.1 & 11.6 & 11.3 & 10.4 & 10.5 & 10.3 & 10.9 & 10.3 \\
\hline 17 & - & - & - & - & 12.3 & 11.7 & 11.1 & 9.8 & 10.8 & 10.3 & 10.9 & 10.3 \\
\hline 18 & - & - & - & - & 12.3 & 11.6 & 10.6 & 9.6 & 10.8 & 10.6 & 11.0 & 10.5 \\
\hline 19 & - & - & - & - & 12.1 & 11.4 & 10.8 & 9.8 & 11.0 & 10.7 & 11.0 & 10.5 \\
\hline 20 & - & - & - & - & 12.0 & 11.1 & 11.0 & 10.4 & 10.8 & 10.5 & 11.1 & 10.5 \\
\hline 21 & - & - & - & - & 11.6 & 10.8 & 11.7 & 10.8 & 10.6 & 10.3 & 11.0 & 10.6 \\
\hline 22 & - & - & - & - & 11.3 & 10.3 & 11.6 & 10.9 & 10.5 & 10.3 & 11.1 & 10.4 \\
\hline 23 & - & - & - & - & 11.1 & 10.2 & 11.3 & 10.8 & 10.6 & 10.2 & 11.2 & 10.6 \\
\hline 31 & - & - & 11.6 & 10.8 & - & - & 11.2 & 11.0 & 10.4 & 10.1 & - & - \\
\hline Month & - & - & - & - & 12.6 & 9.9 & 11.9 & 9.6 & 11.2 & 9.7 & - & - \\
\hline
\end{tabular}


Table 11. Dissolved oxygen data for USGS streamflow-gaging station 15298040, Chulitna River 5 miles above mouth, near Port Alsworth, Alaska, water years 2010 and 2011.-Continued

[Abbreviations: Max, maximum; Min, minimum;-, no data]

\begin{tabular}{|c|c|c|c|c|c|c|c|c|c|c|c|c|}
\hline \multirow{3}{*}{ Day } & \multicolumn{12}{|c|}{ Dissolved oxygen, water, unfiltered, milligrams per liter, water year October 2010 to September 2011} \\
\hline & \multicolumn{2}{|c|}{ April } & \multicolumn{2}{|c|}{ May } & \multicolumn{2}{|c|}{ June } & \multicolumn{2}{|c|}{ July } & \multicolumn{2}{|c|}{ August } & \multicolumn{2}{|c|}{ September } \\
\hline & Max & Min & Max & Min & Max & Min & Max & Min & Max & Min & Max & Min \\
\hline 1 & - & - & - & - & 12.1 & 11.3 & 11.0 & 10.6 & 10.5 & 10.1 & 11.4 & 10.9 \\
\hline 2 & - & - & - & - & 12.2 & 11.5 & 10.8 & 10.3 & 10.8 & 10.3 & 11.1 & 10.7 \\
\hline 3 & - & - & - & - & 11.8 & 11.1 & 11.0 & 10.3 & 11.0 & 10.6 & 11.1 & 10.6 \\
\hline 4 & - & - & - & - & 12.3 & 11.5 & 11.1 & 10.5 & 11.0 & 10.5 & 11.2 & 10.6 \\
\hline 5 & - & - & - & - & 12.3 & 11.8 & 11.2 & 10.7 & 11.5 & 10.9 & 11.0 & 10.4 \\
\hline 6 & - & - & - & - & 12.3 & 11.7 & 10.9 & 10.3 & 11.4 & 11.1 & - & - \\
\hline 7 & - & - & - & - & 12.0 & 11.5 & 10.9 & 10.3 & 11.7 & 11.3 & 11.3 & 10.5 \\
\hline 8 & - & - & - & - & 12.2 & 11.7 & 11.1 & 10.5 & 11.4 & 11.2 & 11.7 & 10.8 \\
\hline 9 & - & - & - & - & 12.0 & 11.4 & 11.1 & 10.6 & 11.6 & 11.2 & 12.2 & 11.2 \\
\hline 10 & - & - & - & - & 11.8 & 11.2 & 11.4 & 10.8 & 11.4 & 11.0 & - & - \\
\hline 11 & - & - & - & - & 11.7 & 10.9 & 11.3 & 11.0 & 11.3 & 10.7 & - & - \\
\hline 12 & - & - & - & - & 11.6 & 10.9 & 11.3 & 10.8 & 11.0 & 10.7 & - & - \\
\hline 13 & - & - & - & - & 11.6 & 10.9 & 11.6 & 11.0 & 11.0 & 10.6 & - & - \\
\hline 14 & - & - & - & - & 11.7 & 10.9 & 11.5 & 11.0 & 10.8 & 10.2 & - & - \\
\hline 15 & - & - & - & - & 11.8 & 11.4 & 11.3 & 10.8 & 10.9 & 10.4 & - & - \\
\hline 16 & - & - & - & - & 11.7 & 11.0 & 11.0 & 10.4 & 10.9 & 10.4 & - & - \\
\hline 17 & - & - & - & - & 11.3 & 10.6 & 10.8 & 10.2 & 10.8 & 10.2 & - & - \\
\hline 18 & - & - & - & - & 11.1 & 10.4 & 11.4 & 10.8 & 10.7 & 10.1 & - & - \\
\hline 19 & - & - & - & - & 11.0 & 10.2 & 11.2 & 10.7 & 10.4 & 9.9 & - & - \\
\hline 20 & - & - & - & - & 11.3 & 10.4 & 11.0 & 10.2 & 10.6 & 9.8 & - & - \\
\hline 21 & - & - & - & - & 11.9 & 11.3 & 10.8 & 10.1 & 10.6 & 9.8 & - & - \\
\hline 22 & - & - & - & - & 11.8 & 11.5 & 10.8 & 10.2 & 10.7 & 9.7 & - & - \\
\hline 23 & - & - & - & - & 11.8 & 11.1 & 10.4 & 9.8 & 11.0 & 10.3 & - & - \\
\hline 24 & - & - & - & - & 11.2 & 10.8 & 11.0 & 10.2 & 11.0 & 10.5 & - & - \\
\hline 25 & - & - & - & - & 11.1 & 10.7 & 11.3 & 10.8 & 11.1 & 10.6 & - & - \\
\hline 26 & - & - & - & - & 11.4 & 10.9 & 11.1 & 10.7 & 11.1 & 10.6 & - & - \\
\hline 27 & - & - & 12.2 & 11.6 & 11.0 & 10.6 & 11.1 & 10.6 & 11.2 & 10.5 & - & - \\
\hline 28 & - & - & 11.8 & 11.2 & 11.3 & 10.9 & 11.1 & 10.5 & 11.1 & 10.6 & - & - \\
\hline 29 & - & - & 12.1 & 11.4 & 11.3 & 11.0 & 10.8 & 10.2 & 11.2 & 10.5 & - & - \\
\hline 30 & - & - & 12.0 & 11.4 & 11.2 & 10.8 & 10.8 & 10.2 & 11.0 & 10.4 & - & - \\
\hline 31 & - & - & 11.7 & 11.0 & - & - & 10.7 & 10.0 & 11.1 & 10.5 & - & - \\
\hline Month & - & - & - & - & 12.3 & 10.2 & 11.6 & 9.8 & 11.7 & 9.7 & - & - \\
\hline
\end{tabular}


Table 12. pH data for USGS streamflow-gaging station 15298040, Chulitna River 5 miles above mouth, near Port Alsworth, Alaska, water years 2010 and 2011.

[Abbreviations: Max, maximum; Min, minimum; -, no data]

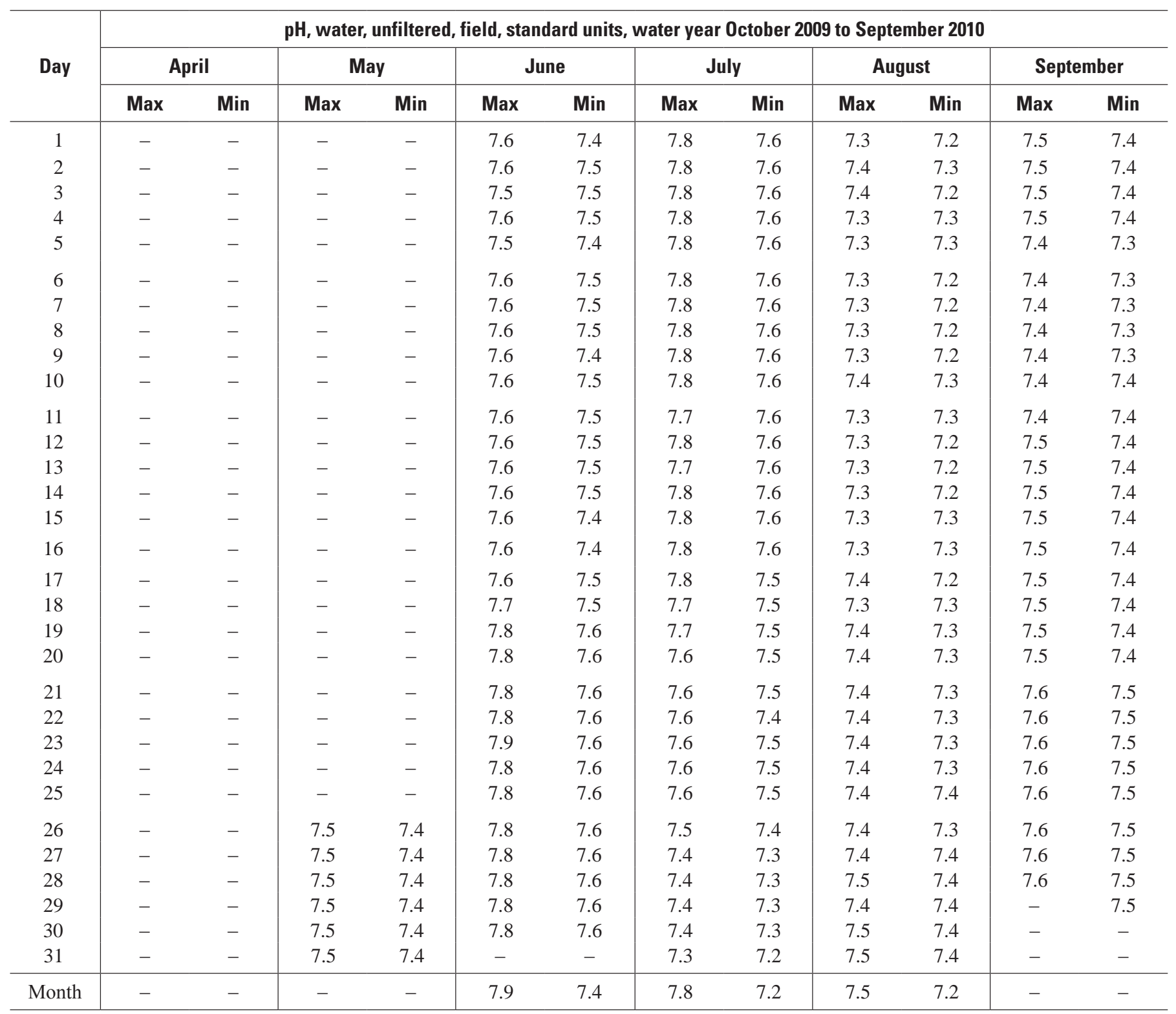


Table 12. pH data for USGS streamflow-gaging station 15298040, Chulitna River 5 miles above mouth, near Port Alsworth, Alaska, water years 2010 and 2011.-Continued

[Abbreviations: Max, maximum; Min, minimum; -, no data]

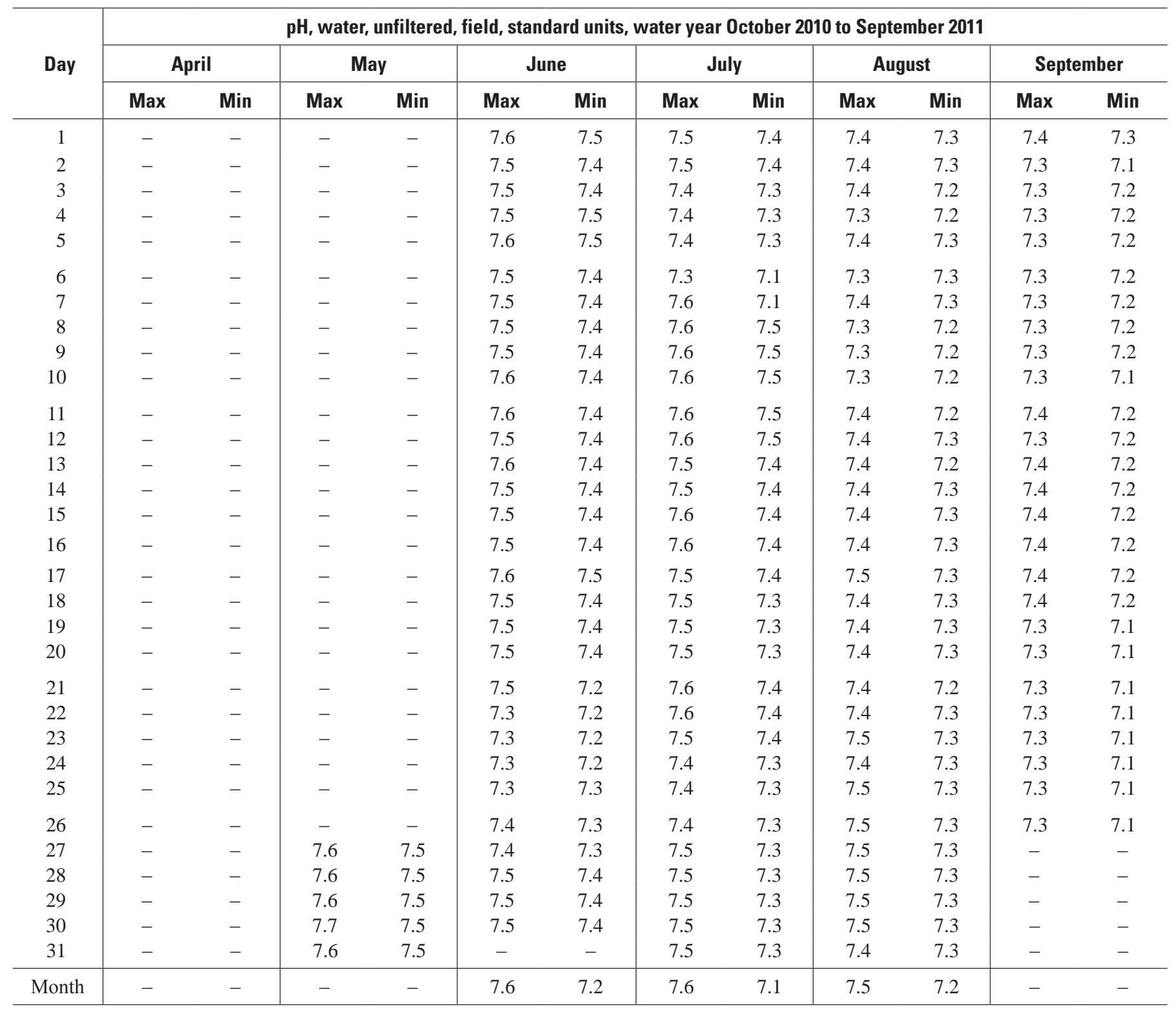


Table 13. Specific conductance data for USGS streamflow-gaging station 15298040, Chulitna River 5 miles above mouth, near Port Alsworth, Alaska, water years 2010 and 2011.

[Abbreviations: Max, maximum; Min, minimum; -, no data]

\begin{tabular}{|c|c|c|c|c|c|c|c|c|c|c|c|c|}
\hline \multirow{3}{*}{ Day } & \multicolumn{12}{|c|}{$\begin{array}{c}\text { Specific conductance, water, unfiltered, microsiemens per centimeter at } 25 \text { degrees Celsius, } \\
\text { water year October } 2009 \text { to September } 2010\end{array}$} \\
\hline & \multicolumn{2}{|c|}{ April } & \multicolumn{2}{|c|}{ May } & \multicolumn{2}{|c|}{ June } & \multicolumn{2}{|c|}{ July } & \multicolumn{2}{|c|}{ August } & \multicolumn{2}{|c|}{ September } \\
\hline & Max & Min & Max & Min & Max & Min & Max & Min & Max & Min & Max & Min \\
\hline 1 & - & - & - & - & 66 & 64 & 80 & 77 & 61 & 59 & 69 & 69 \\
\hline 2 & - & - & - & - & 67 & 66 & 79 & 77 & 62 & 61 & 70 & 69 \\
\hline 3 & - & - & - & - & 67 & 66 & 79 & 78 & 63 & 62 & 71 & 70 \\
\hline 4 & - & - & - & - & 68 & 66 & 79 & 78 & 62 & 61 & 71 & 70 \\
\hline 5 & - & - & - & - & 67 & 65 & 79 & 77 & 61 & 60 & 70 & 69 \\
\hline 6 & - & - & - & - & 68 & 65 & 77 & 74 & 60 & 60 & 70 & 69 \\
\hline 7 & - & - & - & - & 69 & 67 & 75 & 73 & 60 & 59 & 70 & 70 \\
\hline 8 & - & - & - & - & 74 & 69 & 76 & 74 & 60 & 58 & 70 & 69 \\
\hline 9 & - & - & - & - & 74 & 68 & 76 & 76 & 60 & 59 & 70 & 69 \\
\hline 10 & - & - & - & - & 69 & 69 & 77 & 75 & 63 & 60 & 72 & 70 \\
\hline 11 & - & - & - & - & 69 & 68 & 75 & 71 & 61 & 58 & 76 & 72 \\
\hline 12 & - & - & - & - & 70 & 69 & 73 & 71 & 58 & 58 & 75 & 74 \\
\hline 13 & - & - & - & - & 70 & 70 & 74 & 73 & 61 & 58 & 75 & 75 \\
\hline 14 & - & - & - & - & 71 & 70 & 75 & 72 & 61 & 59 & 76 & 75 \\
\hline 15 & - & - & - & - & 71 & 70 & 73 & 72 & 61 & 59 & 76 & 75 \\
\hline 16 & - & - & - & - & 70 & 68 & 73 & 72 & 60 & 59 & 76 & 75 \\
\hline 17 & - & - & - & - & 73 & 69 & 73 & 72 & 59 & 56 & 76 & 75 \\
\hline 18 & - & - & - & - & 72 & 68 & 73 & 72 & 57 & 55 & 77 & 76 \\
\hline 19 & - & - & - & - & 73 & 69 & 72 & 71 & 60 & 57 & 77 & 76 \\
\hline 20 & - & - & - & - & 72 & 68 & 72 & 71 & 62 & 60 & 77 & 77 \\
\hline 21 & - & - & - & - & 72 & 70 & 71 & 70 & 64 & 62 & 78 & 77 \\
\hline 22 & - & - & - & - & 73 & 72 & 73 & 71 & 64 & 63 & 78 & 78 \\
\hline 23 & - & - & - & - & 74 & 73 & 75 & 73 & 64 & 63 & 81 & 78 \\
\hline 24 & - & - & - & - & 76 & 74 & 75 & 74 & 64 & 64 & 79 & 78 \\
\hline 25 & - & - & - & - & 76 & 75 & 75 & 73 & 66 & 64 & 79 & 78 \\
\hline 26 & - & - & 60 & 59 & 77 & 76 & 73 & 64 & 67 & 66 & 78 & 78 \\
\hline 27 & - & - & 60 & 60 & 78 & 76 & 66 & 62 & 68 & 67 & 79 & 78 \\
\hline 28 & - & - & 60 & 58 & 78 & 77 & 67 & 63 & 68 & 68 & 79 & 78 \\
\hline 29 & - & - & 62 & 60 & 79 & 78 & 67 & 65 & 69 & 68 & - & 78 \\
\hline 30 & - & - & 63 & 62 & 79 & 78 & 65 & 60 & 69 & 69 & - & - \\
\hline 31 & - & - & 65 & 63 & - & - & 60 & 59 & 70 & 69 & - & - \\
\hline Month & - & - & - & - & 79 & 64 & 80 & 59 & 70 & 55 & - & - \\
\hline
\end{tabular}


Table 13. Specific conductance data for USGS streamflow-gaging station 15298040, Chulitna River 5 miles above mouth, near Port Alsworth, Alaska, water years 2010 and 2011.-Continued

[Abbreviations: Max, maximum; Min, minimum; -, no data]

\begin{tabular}{|c|c|c|c|c|c|c|c|c|c|c|c|c|}
\hline \multirow{2}{*}{ Day } & \multicolumn{12}{|c|}{$\begin{array}{l}\text { Specific conductance, water, unfiltered, microsiemens per centimeter at } 25 \text { degrees Celsius, } \\
\text { water year October } 2010 \text { to September } 2011\end{array}$} \\
\hline & \multicolumn{2}{|c|}{ April } & \multicolumn{2}{|c|}{ May } & \multicolumn{2}{|c|}{ June } & \multicolumn{2}{|c|}{ July } & \multicolumn{2}{|c|}{ August } & \multicolumn{2}{|c|}{ September } \\
\hline 1 & - & - & - & - & 79 & 76 & 69 & 68 & 74 & 73 & 73 & 71 \\
\hline 2 & - & - & - & - & 81 & 71 & 69 & 69 & 75 & 71 & 73 & 71 \\
\hline 5 & - & - & - & - & 84 & 76 & 67 & 66 & 67 & 65 & 73 & 72 \\
\hline 6 & - & - & - & - & 83 & 77 & 67 & 66 & 70 & 65 & 73 & 72 \\
\hline 7 & - & - & - & - & 83 & 75 & 68 & 67 & 67 & 65 & 73 & 72 \\
\hline 8 & - & - & - & - & 75 & 72 & 69 & 68 & 67 & 65 & 73 & 71 \\
\hline 9 & - & - & - & - & 88 & 74 & 70 & 69 & 65 & 64 & 72 & 70 \\
\hline 13 & - & - & - & - & 76 & 74 & 68 & 68 & 69 & 67 & 72 & 71 \\
\hline 14 & - & - & - & - & 78 & 72 & 70 & 68 & 69 & 68 & 72 & 70 \\
\hline 15 & - & - & - & - & 74 & 72 & 72 & 70 & 73 & 68 & 74 & 71 \\
\hline 16 & - & - & - & - & 77 & 74 & 76 & 71 & 70 & 69 & 73 & 71 \\
\hline 17 & - & - & - & - & 77 & 74 & 73 & 71 & 73 & 70 & 77 & 71 \\
\hline 18 & - & - & - & - & 86 & 75 & 71 & 69 & 71 & 71 & 79 & 74 \\
\hline 19 & - & - & - & - & 86 & 81 & 70 & 69 & 71 & 71 & 77 & 73 \\
\hline 20 & - & - & - & - & 82 & 76 & 72 & 70 & 71 & 69 & 76 & 73 \\
\hline 21 & - & - & - & - & 76 & 61 & 73 & 71 & 71 & 69 & 74 & 72 \\
\hline 22 & - & - & - & - & 61 & 59 & 74 & 73 & 71 & 70 & 74 & 71 \\
\hline 30 & - & - & 73 & 70 & 68 & 67 & 76 & 72 & 75 & 74 & - & - \\
\hline 31 & - & - & 76 & 73 & - & - & 75 & 72 & 75 & 73 & - & - \\
\hline Month & - & - & - & - & 91 & 59 & 76 & 66 & 75 & 64 & - & - \\
\hline
\end{tabular}


Table 14. Turbidity data for USGS streamflow-gaging station 15298040, Chulitna River 5 miles above mouth, near Port Alsworth, Alaska, water years 2010 and 2011.

[Abbreviations: Max, maximum; Min, minimum; -, no data]

\begin{tabular}{|c|c|c|c|c|c|c|c|c|c|c|c|c|}
\hline \multirow{3}{*}{ Day } & \multicolumn{12}{|c|}{$\begin{array}{l}\text { Turbidity, water, unfiltered, near Ir light-emitting diode light, 780-900 nanometers, detect angle } \\
90 \text { degrees formazin nephelometric units (FNU), water year October } 2009 \text { to September } 2010\end{array}$} \\
\hline & \multicolumn{2}{|c|}{ April } & \multicolumn{2}{|c|}{ May } & \multicolumn{2}{|c|}{ June } & \multicolumn{2}{|c|}{ July } & \multicolumn{2}{|c|}{ August } & \multicolumn{2}{|c|}{ September } \\
\hline & Max & Min & Max & Min & Max & Min & Max & Min & Max & Min & Max & Min \\
\hline 1 & - & - & - & - & 6.2 & 3.1 & 4.5 & 3.0 & 12 & 8.6 & - & - \\
\hline 2 & - & - & - & - & 4.6 & 3.0 & 5.3 & 3.0 & 12 & 8.5 & - & - \\
\hline 3 & - & - & - & - & 6.1 & 2.9 & 3.8 & 3.1 & 12 & 8.0 & - & - \\
\hline 4 & - & - & - & - & 6.2 & 3.3 & 4.1 & 3.2 & 20 & 8.6 & 13 & 3.9 \\
\hline 5 & - & - & - & - & 9.9 & 5.2 & 5.0 & 3.2 & 13 & 9.2 & 8.3 & 3.8 \\
\hline 6 & - & - & - & - & 14 & 3.6 & 5.5 & 3.4 & 13 & 8.3 & 6.2 & 3.8 \\
\hline 7 & - & - & - & - & 6.3 & 3.4 & 5.4 & 3.7 & 11 & 7.4 & 6.1 & 3.9 \\
\hline 8 & - & - & - & - & 5.4 & 3.5 & 5.7 & 3.7 & 10 & 7.1 & 8.8 & 4.1 \\
\hline 9 & - & - & - & - & 7.1 & 3.9 & 5.6 & 4.1 & 11 & 6.8 & 7.9 & 4.8 \\
\hline 10 & - & - & - & - & 6.9 & 3.4 & 5.8 & 4.1 & 9.9 & 7.9 & 6.2 & 3.9 \\
\hline 11 & - & - & - & - & 5.0 & 3.0 & 11 & 4.5 & 40 & 8.1 & 5.1 & 3.0 \\
\hline 12 & - & - & - & - & 5.2 & 2.8 & 9.3 & 5.2 & 48 & 10 & 3.6 & 2.3 \\
\hline 13 & - & - & - & - & 5.2 & 3.0 & 6.6 & 5.2 & 16 & 8.8 & 3.0 & 2.3 \\
\hline 14 & - & - & - & - & 11 & 3.1 & 8.4 & 5.6 & 17 & 7.3 & 3.9 & 2.2 \\
\hline 15 & - & - & - & - & 7.2 & 3.3 & 9.1 & 5.9 & 20 & 7.5 & 4.3 & 1.7 \\
\hline 16 & - & - & - & - & 8.4 & 3.9 & 8.6 & 6.2 & 25 & 11 & 2.8 & 1.5 \\
\hline 17 & - & - & - & - & 8.0 & 5.7 & 9.6 & 5.9 & - & 10 & 2.4 & 1.5 \\
\hline 18 & - & - & - & - & 9.2 & 5.9 & 8.7 & 5.8 & - & - & 2.3 & 1.0 \\
\hline 19 & - & - & - & - & 7.4 & 5.4 & 8.8 & 5.9 & - & - & 3.2 & 0.8 \\
\hline 20 & - & - & - & - & 9.3 & 5.8 & 10 & 5.9 & - & - & 3.0 & 0.7 \\
\hline 21 & - & - & - & - & 7.2 & 4.8 & 12 & 7.9 & - & - & 2.4 & 0.6 \\
\hline 22 & - & - & - & - & 8.0 & 3.9 & 8.9 & 5.1 & - & - & 2.4 & 0.9 \\
\hline 23 & - & - & - & - & 5.2 & 3.3 & 6.7 & 4.4 & - & - & 3.2 & 1.3 \\
\hline 24 & - & - & - & - & 5.1 & 3.5 & 8.2 & 4.7 & - & - & 4.2 & 1.5 \\
\hline 25 & - & - & - & - & 5.2 & 3.2 & 7.3 & 4.9 & - & - & 2.8 & 1.3 \\
\hline 26 & - & - & 5.2 & 3.8 & 4.5 & 2.8 & 23 & 5.3 & - & - & 4.2 & 2.3 \\
\hline 27 & - & - & 4.6 & 3.3 & 3.8 & 2.7 & 20 & 10 & - & - & 7.0 & 3.8 \\
\hline 28 & - & - & 5.1 & 3.6 & 4.8 & 2.8 & 11 & 7.1 & - & - & 12 & 6.5 \\
\hline 29 & - & - & 5.4 & 3.8 & 4.2 & 2.9 & 10 & 6.8 & - & - & - & - \\
\hline 30 & - & - & 5.4 & 3.6 & 4.3 & 2.9 & 15 & 7.8 & - & - & - & - \\
\hline 31 & - & - & 5.8 & 3.1 & - & - & 17 & 9.7 & - & - & - & - \\
\hline Month & - & - & - & - & 14 & 2.7 & 23 & 3.0 & - & - & - & - \\
\hline
\end{tabular}


Table 14. Turbidity data for USGS streamflow-gaging station 15298040, Chulitna River 5 miles above mouth, near Port Alsworth, Alaska, water years 2010 and 2011.-Continued

[Abbreviations: Max, maximum; Min, minimum; -, no data]

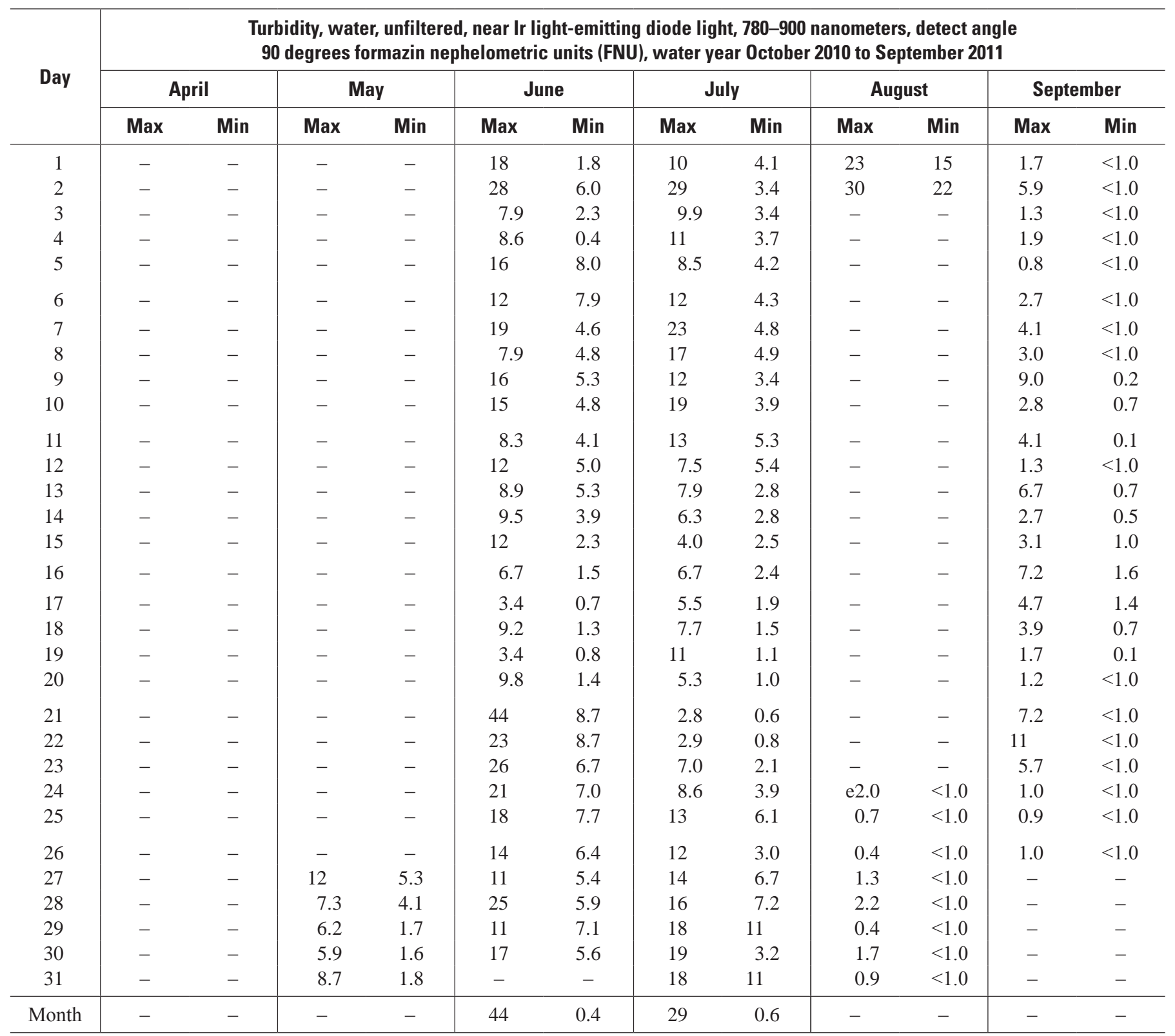


Table 15. Discharge data for USGS streamflow-gaging station 15298040, Chulitna River 5 miles above mouth, near Point Alsworth, Alaska, water years 2010 and 2011.

[Abbreviations: Max, maximum; Min, minimum; e, estimated; Acre-ft, acre-foot; Cfsm, cubic foot per second per square mile; In., inch]

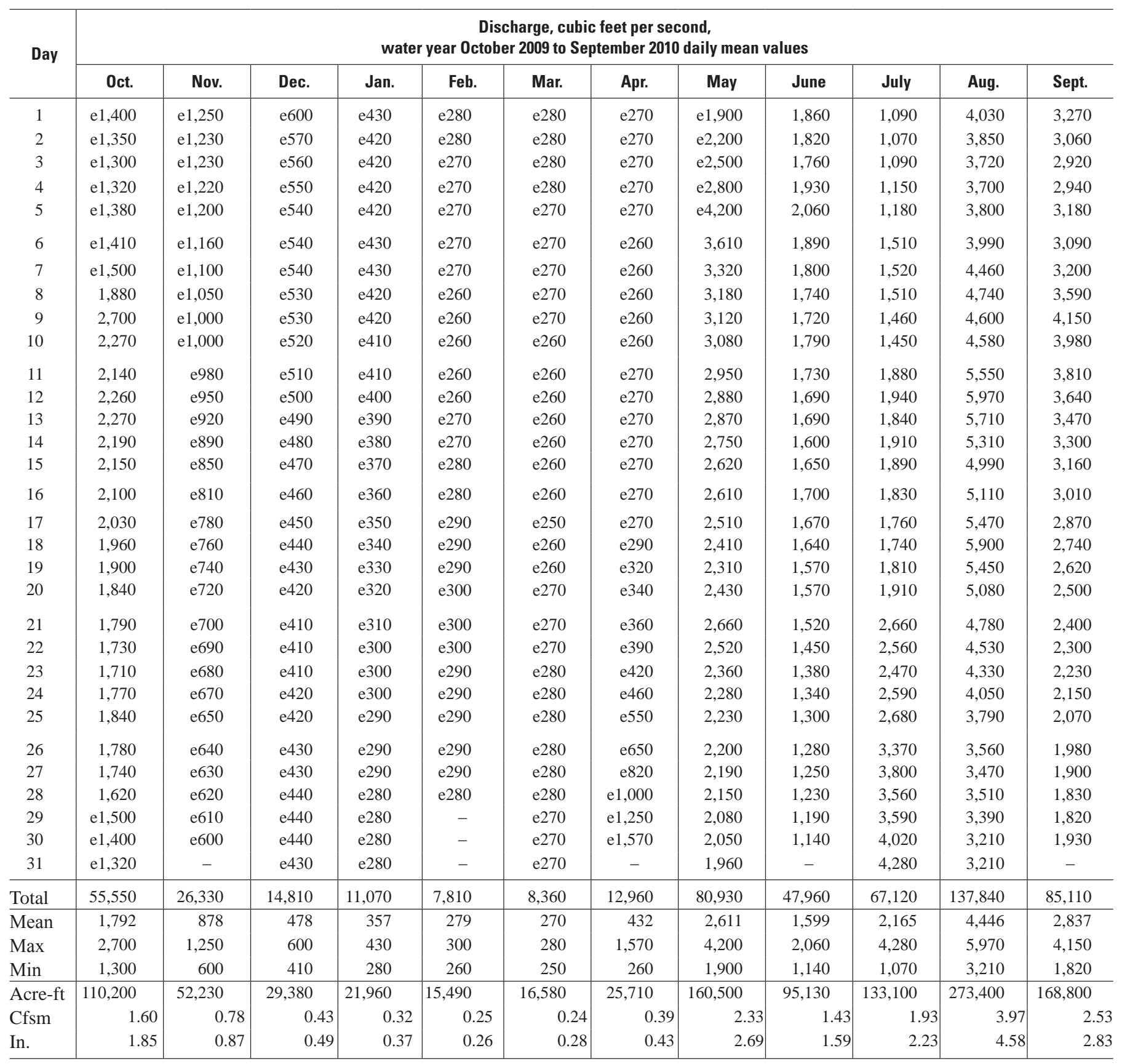


Table 15. Discharge data for USGS streamflow-gaging station 15298040, Chulitna River 5 miles above mouth, near Point Alsworth, Alaska, water years 2010 and 2011. - Continued

[Abbreviations: Max, maximum; Min, minimum; e, estimated; Acre-ft, acre-foot; Cfsm, cubic foot per second per square mile; In., inch]

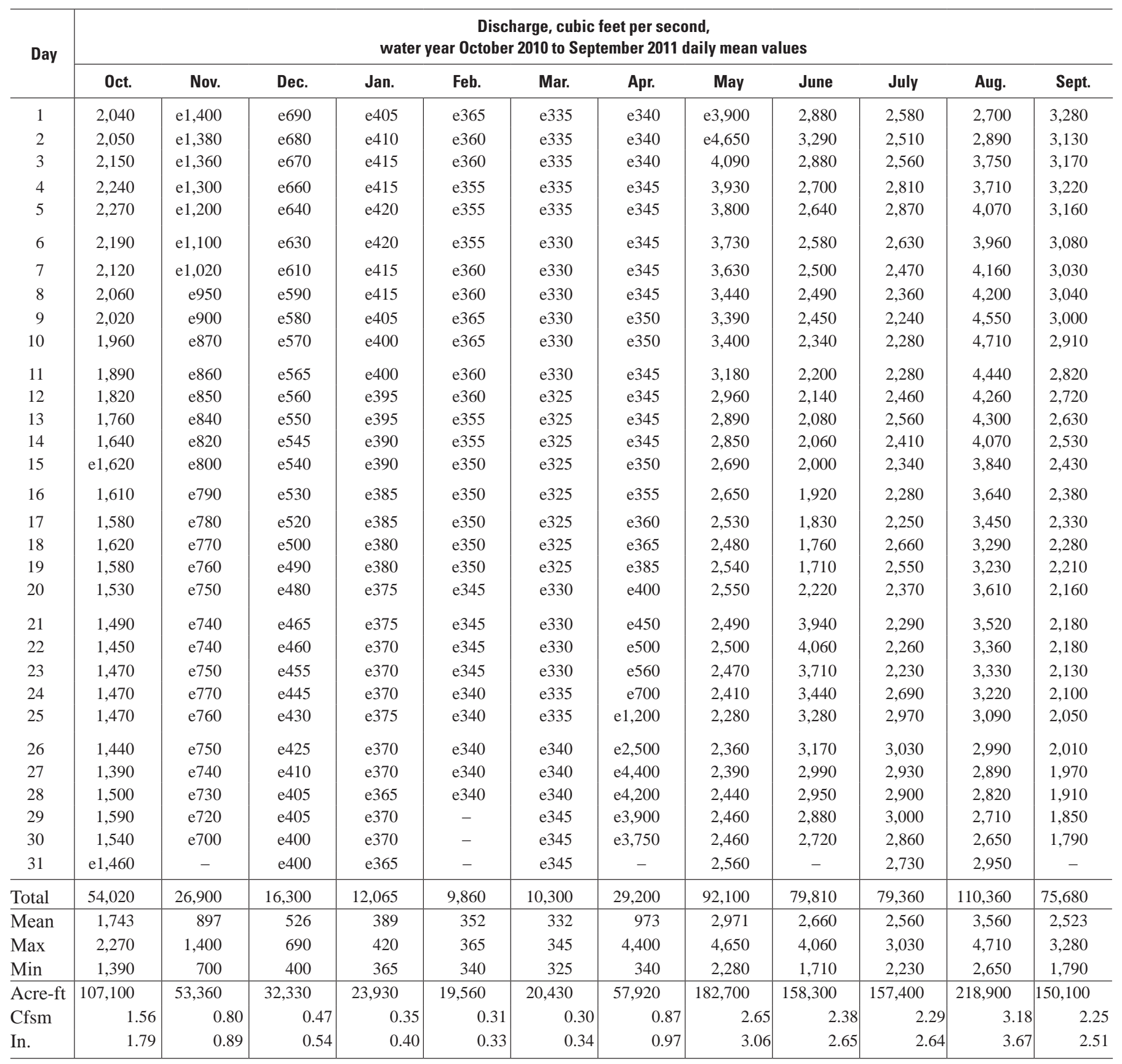


Publishing support provided by the U.S. Geological Survey Publishing Network, Tacoma Publishing Service Center

For more information concerning the research in this report, contact the

Director, Alaska Science Center

U.S. Geological Survey

4210 University Dr.

Anchorage, Alaska 99508-4560

http://alaska.usgs.gov 


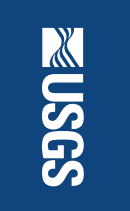

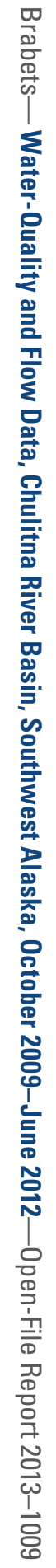

